\title{
Generalized $\alpha$-variation and Lebesgue equivalence to differentiable functions
}

\author{
by \\ Jakub Duda (Rehovot and New York)
}

\begin{abstract}
We find conditions on a real function $f:[a, b] \rightarrow \mathbb{R}$ equivalent to being Lebesgue equivalent to an $n$-times differentiable function $(n \geq 2)$; a simple solution in the case $n=2$ appeared in an earlier paper. For that purpose, we introduce the notions of $C B V G_{1 / n}$ and $S B V G_{1 / n}$ functions, which play analogous rôles for the $n$th order differentiability to the classical notion of a $V B G_{*}$ function for the first order differentiability, and the classes $C B V_{1 / n}$ and $S B V_{1 / n}$ (introduced by Preiss and Laczkovich) for $C^{n}$ smoothness. As a consequence, we deduce that Lebesgue equivalence to an $n$-times differentiable function is the same as Lebesgue equivalence to a function $f$ which is $(n-1)$-times differentiable with $f^{(n-1)}(\cdot)$ pointwise Lipschitz. We also characterize functions that are Lebesgue equivalent to $n$-times differentiable functions with a.e. nonzero derivatives. As a corollary, we establish a generalization of Zahorski's Lemma for higher order differentiability.
\end{abstract}

1. Introduction. Let $f:[a, b] \rightarrow \mathbb{R}$. We say that $f$ is Lebesgue equivalent to $g:[a, b] \rightarrow \mathbb{R}$ provided there exists a homeomorphism $h$ of $[a, b]$ onto itself such that $g=f \circ h$. This terminology is taken from [3]. Zahorski [15] and Choquet [4] (see also Tolstov [14]) characterized paths $f:[a, b] \rightarrow \mathbb{R}^{n}$ that allow a differentiable parametrization (resp. a differentiable parametrization with almost everywhere nonzero derivative) as those paths that have the $V B G_{*}$ property (resp. which are also not constant on any interval). Fleissner and Foran [9] reproved this later (for real functions only and not considering the case of a.e. nonzero derivatives) using a different result of Tolstov. The definition of $V B G_{*}$ is classical; see e.g. [13]. The above mentioned results were generalized by L. Zajíček and the author [6] to paths with values in Banach spaces (and also metric spaces using the metric derivative instead of the usual one). Laczkovich and Preiss [11] and Lebedev [12] studied (among other things) the case of $C^{n}$-parametrizations of real-valued functions $(n \geq 2)$. Lebedev proved that a continuous function $f:[a, b] \rightarrow \mathbb{R}$ is

2010 Mathematics Subject Classification: Primary 26A24; Secondary 26A45.

Key words and phrases: differentiability, Zahorski's Lemma, higher order differentiability, differentiability via homeomorphisms. 
Lebesgue equivalent to a $C^{n}$ function provided

$$
\lambda\left(f\left(K_{f}\right)\right)=0 \text { and } \sum_{\alpha \in A}\left(\omega_{\alpha}^{f}\right)^{1 / n}<\infty,
$$

where $K_{f}$ is the set of point of varying monotonicity of $f$ (see the definition below) and $\omega_{\alpha}^{f}$ is the oscillation of $f$ on $I_{\alpha}$, where $\left(I_{\alpha}\right)_{\alpha \in A}$ are all the intervals contiguous to $K_{f}$ in $[a, b]$. Laczkovich and Preiss showed that the same is true for a continuous $f$ provided

$$
V_{1 / n}\left(f, K_{f}\right)<\infty
$$

or

$$
S V_{1 / n}\left(f, K_{f}\right)=0 \text {. }
$$

(See Definition 2.3 in Section 2). They define $C B V_{1 / n}\left(\right.$ resp. $\left.S B V_{1 / n}\right)$ as the class of continuous function which satisfy (1.1) (resp. (1.2)). Moreover, in [11] and [12] also the case of $C^{n, \alpha}(0<\alpha \leq 1)$ parametrizations is settled (where $C^{n, \alpha}$ is the class of functions such that $f^{(n)}$ is $\alpha$-Hölder).

Differentiability via a homeomorphic change of variable was studied by other authors (see e.g. [2], [1]). For a nice survey of differentiability of real-valued functions via homeomorphisms, see [10]. L. Zajičcek and the author $[7,8]$ characterized Banach space-valued paths (for Banach spaces with a $C^{1}$ norm) admitting a $C^{2}$-parametrization or a parametrization with finite convexity. In the corresponding situations, also the case of the first derivative being almost everywhere nonzero is treated in $[7,8]$.

In [5], we characterized functions $f:[a, b] \rightarrow \mathbb{R}$ Lebesgue equivalent to twice differentiable functions. We introduced the notion of $V B G_{1 / 2}$ functions for that purpose. We also established that for a real function $f$ defined on a closed interval, being Lebesgue equivalent to a twice differentiable function is equivalent to being Lebesgue equivalent to a differentiable function whose derivative is pointwise Lipschitz.

In the present article, we characterize functions Lebesgue equivalent to $n$-times differentiable functions for $n \geq 3$ (our present approach gives a certain condition also in case $n=2$, which can be seen to be equivalent to the one proved in [5], but the general proof is much more complicated than the arguments of [5] in that interesting special case). We introduce two new classes of functions: $C B V G_{1 / n}$ and $S B V G_{1 / n}$, analogous to the classes $C B V_{1 / n}$ and $S B V_{1 / n}$ introduced by Preiss and Laczkovich in [11] to characterize functions Lebesgue equivalent to $C^{n}$ functions. In the main Theorem 4.1, we prove that $f$ is Lebesgue equivalent to an $n$-times differentiable function if and only if $f$ is $C B V G_{1 / n}$ (resp. $f$ is $S B V G_{1 / n}$ ). As a corollary, we deduce that the classes $C B V G_{1 / n}$ and $S B V G_{1 / n}$ coincide (which seems to be difficult to establish directly). Our approach also shows that $f$ is Lebesgue equivalent to an $n$-times differentiable function if and only 
if $f$ is Lebesgue equivalent to an $(n-1)$-times differentiable function $g$ such that $g^{(n-1)}(\cdot)$ is pointwise Lipschitz (see Theorem 4.1). This corresponds to the analogous situation for $n=2$ in [5], and is similar to the phenomenon that $f:[a, b] \rightarrow \mathbb{R}$ is Lebesgue equivalent to a $C^{n}$ function if and only if $f$ is Lebesgue equivalent to a $C^{(n-1), 1}$ function or a function which has bounded $n$th derivative (see [11, Remark 3.7]), which is proved in [11]. We also present an example (see Example 4.3) which shows that for each $n \geq 2$ there exists a continuous function which is $C B V G_{1 / n}$, but not Lebesgue equivalent to any $C^{n}$ function. In Theorem 4.4, we characterize Lebesgue equivalence to an $n$-times differentiable function whose first derivative is a.e. nonzero.

The classical Zahorski Lemma (see e.g. [15] or [10, p. 27]) states that if $M \subset[a, b]$ has Lebesgue measure zero, then there exists a (boundedly) differentiable homeomorphism $h$ from $[a, b]$ onto itself such that $h^{-1}(M) \subset$ $\left\{x \in[a, b]: h^{\prime}(x)=0\right\}$. If $M$ is closed, then $h$ can be taken $C^{1}$. In Theorem 5.1, we show a higher order analogue of this fact: a closed set $M \subset[a, b]$ is the image of an $n$-times differentiable homeomorphism of $[a, b]$ such that $h^{(i)}(x)=0$ for all $x \in h^{-1}(M)$ and $i=1, \ldots, n$ if and only if there exists a decomposition of $M$ such that certain variational conditions closely related to the definition of the class $C B V G_{1 / n}$ (respectively, $S B V G_{1 / n}$ ) are satisfied. See Theorem 5.1 for details.

The current paper is structured as follows. Section 2 contains basic facts and definitions. Section 3 contains facts about the generalized variation $G V_{1 / n}$ (and related notions) and classes $C B V G_{1 / n}$, resp. $S B V G_{1 / n}$; there is also the definition of an auxiliary class $\overline{S B V G}_{1 / n}$. Section 4 contains the main Theorems 4.1 and 4.4. In Section 5 we prove Theorem 5.1, which is an analogue of Zahorski's Lemma for higher order differentiability.

In the proofs, we need many auxiliary results. Let us point out that the main ingredients for our results are the estimate of Lemma 3.8, and the method of construction of a suitable variation in Lemmata 3.10 and 3.11.

2. Preliminaries. By $C$ (resp. $C_{x}, \ldots$ ) we will denote an absolute constant (resp. constant depending on $x, \ldots$ ) that can change from line to line. By $n$ we always denote a positive integer. By $\lambda$ we denote the Lebesgue measure on $\mathbb{R}$. For $x, r \in \mathbb{R}$ with $r>0$ we denote by $B(x, r):=\{y \in \mathbb{R}:|x-y|$ $<r\}$ the open ball with centre $x$ and radius $r$. Let $K \subset[a, b]$ be closed and such that $\{a, b\} \subset K$. We say that the interval $(c, d) \subset[a, b]$ is contiguous to $K($ in $[a, b])$ provided $c, d \in K$ and $(c, d) \cap K=\emptyset$ (i.e. it is a maximal open component of $[a, b] \backslash K$ in $[a, b])$. Let $f:[a, b] \rightarrow \mathbb{R}$. By $K_{f}$ we will denote the set of points of varying monotonicity of $f$, i.e. of $x \in[a, b]$ such that there is no open neighbourhood $U$ of $x$ such that $\left.f\right|_{U}$ is either constant or strictly monotone (see e.g. [11]). Obviously, $K_{f}$ is closed and $\{a, b\} \subset K_{f}$. 
We will also frequently use the simple fact that if $h$ is a homeomorphism of $[a, b]$ onto itself, and $g=f \circ h$, then $K_{g}=h^{-1}\left(K_{f}\right)$.

Let $f:[a, b] \rightarrow \mathbb{R}$. We say that $f$ is pointwise Lipschitz at $x \in[a, b]$ provided

$$
\limsup _{\substack{t \rightarrow 0 \\ x+t \in[a, b]}} \frac{|f(x+t)-f(x)|}{|t|}<\infty .
$$

We say that $f$ is pointwise Lipschitz provided it is pointwise Lipschitz at each $x \in[a, b]$. We define the derivative $f^{\prime}(x)$ of $f$ at $x \in[a, b]$ and the $n$th derivative $f^{(n)}(x), n \geq 0$, as usual; at the endpoints we consider the corresponding unilateral derivatives. We say that $f$ is $C^{n}$ for $n \geq 1$ provided $f^{(n)}$ exists and is continuous in $[a, b]$. We will often use the following easy fact: if $f$ is $C^{1}$ and $x \in K_{f} \cap(a, b)$, then $f^{\prime}(x)=0$.

The following version of Sard's theorem is proved e.g. in [6, Lemma 2.2].

Lemma 2.1. If $f: \mathbb{R} \rightarrow \mathbb{R}$, then $\lambda\left(f\left(\left\{x \in \mathbb{R}: f^{\prime}(x)=0\right\}\right)\right)=0$.

The following simple lemma is proved in [5, Lemma 9].

Lemma 2.2. Let $h_{m}:[a, b] \rightarrow\left[c_{m}, d_{m}\right](m \in \mathcal{M} \subset \mathbb{N})$ be continuous increasing functions such that $\sum_{m \in \mathcal{M}} h_{m}(x) \in \mathbb{R}$ for all $x \in[a, b]$. Let $K \subset[a, b]$ be closed and such that $\lambda\left(h_{m}(K)\right)=0$ for all $m \in \mathcal{M}$. Then $h:[a, b] \rightarrow[c, d]$, defined as $h(x):=\sum_{m \in \mathcal{M}} h_{m}(x)$, is a continuous and increasing function (for some $c, d \in \mathbb{R}$ ) such that $\lambda(h(K))=0$.

The following definition is taken from [11].

Definition 2.3. Let $f:[a, b] \rightarrow \mathbb{R}$ be continuous. For $\alpha \in(0,1], \delta>0$, and $K \subset[a, b]$, we define $V_{\alpha}^{\delta}(f, K)$ as the supremum of the sums

$$
\sum_{i=1}^{N}\left|f\left(d_{i}\right)-f\left(c_{i}\right)\right|^{\alpha},
$$

where $\left(\left[c_{i}, d_{i}\right]\right)_{i=1}^{N}$ is any sequence of nonoverlapping intervals in $[a, b]$ such that $c_{i}, d_{i} \in K$ and $d_{i}-c_{i} \leq \delta$ for all $i=1, \ldots, N$. We define $V(f,[a, b]):=$ $V_{1}^{b-a}(f,[a, b]), V_{\alpha}(f, K):=V_{\alpha}^{b-a}(f, K)$, and

$$
S V_{\alpha}(f, K):=\lim _{\delta \rightarrow 0+} V_{\alpha}^{\delta}(f, K) .
$$

See the paper [11] for basic properties of these fractional variations. Note that [11, Lemma 3.13] implies that if $K \subset[a, b]$ is closed and $V_{\alpha}^{\delta}(f, K)<\infty$, then the function $g(x)=V_{\alpha}^{\delta}(f, K \cap[a, x])$ is continuous on $K$.

We have the following simple lemma.

Lemma 2.4. Suppose that $H \subset \mathbb{R}$ is bounded, $f: H \rightarrow \mathbb{R}$ is uniformly continuous and $V_{\alpha}^{\delta}(f, H)<\infty$ for some $\alpha \in(0,1)$ and $\delta>0$. Then $\lambda(\overline{f(H)})=0$. 
Proof. Let $\varepsilon>0$ and put

$$
\eta:=\left(\frac{\varepsilon}{V_{\alpha}^{\delta}(f, H)+1}\right)^{1 /(1-\alpha)} .
$$

Choose $0<\zeta<\delta$ such that for all $x, y \in H$ with $|x-y|<\zeta$ we have $|f(x)-f(y)|<\eta$. Let $\left(\left[a_{i}, b_{i}\right]\right)_{i=1}^{N}$ be nonoverlapping intervals with $a_{i}, b_{i} \in H$ and $b_{i}-a_{i}<\zeta$. Then

$$
\sum_{i=1}^{N}\left|f\left(b_{i}\right)-f\left(a_{i}\right)\right| \leq \eta^{1-\alpha} V_{\alpha}^{\delta}(f, H)<\varepsilon .
$$

Thus we have $S V_{1}(f, H)=0$; [11, Theorem 2.9] implies $\lambda(\overline{f(H)})=0$.

We shall need the following lemma. For a proof, see e.g. [6, Lemma 2.7].

Lemma 2.5. Let $\{a, b\} \subset B \subset[a, b]$ be closed, and $f:[a, b] \rightarrow \mathbb{R}$ be continuous. If $\lambda(f(B))=0$, then $V(f,[a, b])=\sum_{i \in \mathcal{I}} V\left(f,\left[c_{i}, d_{i}\right]\right)$, where $I_{i}=\left(c_{i}, d_{i}\right)(i \in \mathcal{I} \subset \mathbb{N})$ are all intervals contiguous to $B$ in $[a, b]$.

Lemma 2.6. Let $f:[a, b] \rightarrow \mathbb{R}$ be continuous, and $\{a, b\} \subset K \subset[a, b]$ be closed such that $V_{\alpha}^{\delta}(f, K)<\infty$ for some $\alpha \in(0,1)$ and $\delta>0$, and $V(f,[c, d])=|f(d)-f(c)|$ whenever $(c, d)$ is an interval contiguous to $K$ in $[a, b]$. Then $V(f,[a, b])<\infty$.

Proof. Let $\left(u_{p}, v_{p}\right)(p \in \mathcal{P} \subset \mathbb{N})$ be all the intervals contiguous to $K$ in $[a, b]$. By Lemma 2.4, we have $\lambda(f(K))=0$, and thus by Lemma 2.5 and the assumptions, we obtain

$V(f,[a, b])=\sum_{p \in \mathcal{P}} V\left(f,\left[u_{p}, v_{p}\right]\right)=\sum_{p \in \mathcal{P}}\left|f\left(v_{p}\right)-f\left(u_{p}\right)\right| \leq 2 N M+V_{\alpha}^{\delta}(f, K)<\infty$,

where $N$ is the number of $p \in \mathcal{P}$ such that either $\left|f\left(v_{p}\right)-f\left(u_{p}\right)\right|>1$ or $v_{p}-u_{p} \geq \delta$ (which is finite since $V_{\alpha}^{\delta}(f, K)<\infty$ and $b-a<\infty$ ), and $M:=\max _{x \in[a, b]}|f(x)|$.

Lemma 2.7. Let $f:[a, b] \rightarrow \mathbb{R}$ be continuous, $\{a, b\} \subset K \subset[a, b]$ closed, $V_{\alpha}^{\delta}(f, K)<\infty$ for some $\alpha \in(0,1)$ and $\delta>0$, and $V(f,[u, v])=|f(v)-f(u)|$ whenever $(u, v)$ is an interval contiguous to $K$. Let $g(x):=V_{\alpha}^{\delta}(f, K \cap[a, x])$. Then $\lambda(g(K))=0$.

Proof. Lemma 2.4 shows that $\lambda(f(K))=0$, and by Lemma 2.6 it follows that $V(f,[a, b])<\infty$. Let $\tilde{g}(x)=g(x)$ for $x \in K$. Now, [11, Lemma 3.13] shows that $g$ is continuous on $K$. Extend $\tilde{g}$ to $[a, b]$ in such a way that $\tilde{g}$ is affine and continuous on $[u, v]$ whenever $(u, v)$ is an interval contiguous to $K$. Since $g(K)=\tilde{g}(K)$, it is enough to prove that $\lambda(\tilde{g}(K))=0$. Let $\varepsilon>0$ and $\left(\left[c_{i}, d_{i}\right]\right)_{i=1}^{N}$ be nonoverlapping intervals with $c_{i}, d_{i} \in K, d_{i}-c_{i} \leq \delta$ for each $i=1, \ldots, N$, and $V_{\alpha}^{\delta}(f, K) \leq \sum_{i=1}^{N}\left|f\left(d_{i}\right)-f\left(c_{i}\right)\right|^{\alpha}+\varepsilon / 2$. For each 
$i=1, \ldots, N$, by Lemma 2.5 find intervals $\left(\left[c_{j}^{i}, d_{j}^{i}\right]\right)_{j=1}^{J_{i}}$ contiguous to $K$ in $\left[c_{i}, d_{i}\right]$ and such that

$$
\left|f\left(d_{i}\right)-f\left(c_{i}\right)\right| \leq V\left(f,\left[c_{i}, d_{i}\right]\right) \leq \sum_{j=1}^{J_{i}}\left|f\left(d_{j}^{i}\right)-f\left(c_{j}^{i}\right)\right|+\left(\frac{\varepsilon}{2 N}\right)^{1 / \alpha} .
$$

Thus

$$
\tilde{g}(b)-\tilde{g}(a)=V_{\alpha}^{\delta}(f, K) \leq \sum_{i=1}^{N} \sum_{j=1}^{J_{i}}\left|f\left(d_{j}^{i}\right)-f\left(c_{j}^{i}\right)\right|^{\alpha}+\varepsilon \leq \sum_{p \in \mathcal{P}}\left|f\left(v_{p}\right)-f\left(u_{p}\right)\right|^{\alpha}+\varepsilon,
$$

where $\left(u_{p}, v_{p}\right)(p \in \mathcal{P} \subset \mathbb{N})$ are all the intervals contiguous to $K$ in $[a, b]$. Now, send $\varepsilon \rightarrow 0+$ to conclude that $\tilde{g}(d)-\tilde{g}(c) \leq \sum_{p \in \mathcal{P}}\left(\tilde{g}\left(v_{p}\right)-\tilde{g}\left(u_{p}\right)\right)$. Since $\tilde{g}(K) \cap \tilde{g}\left(\bigcup_{p \in \mathcal{P}}\left(u_{p}, v_{p}\right)\right)$ is countable, we obtain

$$
\lambda(\tilde{g}(K))=(\tilde{g}(b)-\tilde{g}(a))-\sum_{p \in \mathcal{P}}\left(\tilde{g}\left(v_{p}\right)-\tilde{g}\left(u_{p}\right)\right)=0 .
$$

REMARK 2.8. Lemmata 2.2 and 2.7 together with the methods from [11] can be used to prove the following: Suppose that $f:[a, b] \rightarrow \mathbb{R}$ is continuous, and $n \geq 2$. Then the following are equivalent:

(i) $f$ is Lebesgue equivalent to a $C^{n}$ function $g$ such that $g^{\prime}(x) \neq 0$ for almost all $x \in[a, b]$;

(ii) $V_{1 / n}\left(f, K_{f}\right)<\infty$ and $f$ is not constant on any interval;

(iii) $S V_{1 / n}\left(f, K_{f}\right)=0$ and $f$ is not constant on any interval.

See Definition 2.3 for the definitions of the fractional variations. In (i), we can actually replace " $C$ " " with " $C$ ", where $s>1$ (we did not define this class) if we replace $1 / n$ by $1 / s$ in the variations in (ii) and (iii). See [11, Definition 3.1] for the definition of this class.

Let $K \subset \mathbb{R}$ be closed. As usual, we denote by $K^{\prime}$ the set of all accumulation points of $K$. It is easy to see that $K^{\prime}$ is closed and $K \backslash K^{\prime}$ is countable.

We have the following easy consequence of the classical Rolle theorem.

Lemma 2.9. Let $f:[a, b] \rightarrow \mathbb{R}$ be $m$-times differentiable for some $m \geq 1$, and suppose that there are $m$ distinct points $\left(x_{i}\right)_{i=1}^{m}$ in $[a, b]$ such that $f^{\prime}\left(x_{i}\right)$ $=0$ for $i=1, \ldots, m$. Then there exists $y \in\left[\min _{1 \leq i \leq m} x_{i}, \max _{1 \leq i \leq m} x_{i}\right]$ such that $f^{(m)}(y)=0$.

The following lemma shows that the derivatives are zero at all accumulation points of a given set.

Lemma 2.10. Let $f:[a, b] \rightarrow \mathbb{R}$ be $(n-1)$-times differentiable for some $n \geq 2$, and let $K \subset\left\{x \in[a, b]: f^{\prime}(x)=0\right\}$ be a closed set. Then $f^{(i)}(x)=0$ whenever $x \in K^{\prime} \cap(a, b)$ and $i \in\{1, \ldots, n-1\}$. If $x \in K^{\prime} \cap(a, b)$ and $f^{(n)}(x)$ exists, then $f^{(n)}(x)=0$. 
Proof. By assumptions, we have $f^{\prime}(x)=0$ for all $x \in K$. Let $i \in$ $\{2, \ldots, n-1\}$. Without any loss of generality, assume that $x$ is a right-hand accumulation point of $K$. Fix $k \in \mathbb{N}$. There are $x<z_{1}<\cdots<z_{i-1}<x+1 / k$ with $z_{j} \in K$. By Lemma 2.9 (with $m=i-1$ ) there exists $w_{k} \in\left[z_{1}, z_{i-1}\right]$ with $f^{(i-1)}\left(w_{k}\right)=0$, and thus (by induction) we have

$$
f^{(i)}(x)=\lim _{k \rightarrow \infty} \frac{f^{(i-1)}\left(w_{k}\right)}{w_{k}-x}=0 \quad \text { for } i=2, \ldots, n-1 .
$$

If $f^{(n)}(x)$ exists, then the argument above implies that it is equal to 0 .

The next lemma will allow us to construct suitable extensions of functions.

Lemma 2.11. Let $\alpha, \beta, A, B \in \mathbb{R}$ with $\alpha<\beta, A<B, n \in \mathbb{N}, n \geq 2$. Then there exists an $n$-times differentiable homeomorphism $H:[\alpha, \beta] \rightarrow[A, B]$ such that

(i) $H^{(i)}(\alpha)=H^{(i)}(\beta)=0$ for $i=1, \ldots, n, H^{\prime}(x)>0$ for all $x \in(\alpha, \beta)$,

(ii) $H(\alpha)=A, H(\beta)=B$,

(iii) $\left|H^{(n-1)}(t)\right| \leq C \cdot \frac{|B-A|}{(\beta-\alpha)^{n}} \cdot \min (t-\alpha, \beta-t)$ for $t \in(\alpha, \beta)$, where $C>0$ is an absolute constant.

Proof. As in [11, p. 420], denote

$$
w(x)=c \quad \exp \left(-t^{-2}-(1-t)^{-2}\right) d t \quad(x \in[0,1])
$$

where the positive constant $c$ is chosen such that $w(1)=1$. Then $w \in$ $C^{\infty}([0,1]), w(0)=0, w$ is strictly increasing on $[0,1], w^{\prime}(x) \neq 0$ for all $x \in(0,1)$, and $w^{(i)}(0)=w^{(i)}(1)=0$ for $i=1,2, \ldots$ For $x \in[\alpha, \beta]$ define

$$
H(x)=A+(B-A) \cdot w\left(\frac{x-\alpha}{\beta-\alpha}\right) .
$$

It is easy to see that the conditions (i) and (ii) hold. Condition (iii) follows from the fact that $w$ is $C^{\infty}$ and thus $H^{(n-1)}(\cdot)$ is $C|B-A| /(\beta-\alpha)^{n_{-}}$ Lipschitz on $[\alpha, \beta]$.

3. Generalized fractional variation. We need the following generalized fractional variation.

Definition 3.1. Let $f:[a, b] \rightarrow \mathbb{R}$. Let $\emptyset \neq A \subset K$ be closed sets. We define the generalized $1 / n$-variation $G V_{1 / n}(f, A, K)$ (resp. $\overline{G V}_{1 / n}^{\delta}(f, A, K)$ for $\delta>0$ ) as the supremum of the sums

$$
\sum_{i=1}^{N}\left(V_{1 /(n-1)}\left(f, K \cap\left[x_{i}, y_{i}\right]\right)\right)^{(n-1) / n}
$$


over all collections $\left(\left[x_{i}, y_{i}\right]\right)_{i=1}^{N}$ of nonoverlapping intervals in $[a, b]$ with $x_{i}, y_{i}$ $\in A$ for all $i=1, \ldots, N$ (resp. over all collections $\left(\left[x_{i}, y_{i}\right]\right)_{i=1}^{N}$ of nonoverlapping intervals in $[a, b]$ such that $y_{i}-x_{i} \leq \delta, x_{i}, y_{i} \in K$, and $\left\{x_{i}, y_{i}\right\} \cap A \neq \emptyset$ for all $i=1, \ldots, N)$.

We put $G V_{1 / n}(f, \emptyset, K)=\overline{G V}_{1 / n}^{\delta}(f, \emptyset, K)=0$. Similarly, we also define the auxiliary variation $r G V_{1 / n}^{\delta}(f, A, K)$ (resp. $\left.l G V_{1 / n}^{\delta}(f, A, K)\right)$ as the supremum of the sums in (3.1) for $\overline{G V}_{1 / n}^{\delta}$, but requiring that $x_{i} \in A$ (resp. $y_{i} \in A$ ) for all $i=1, \ldots, N$ whenever $\left(\left[x_{i}, y_{i}\right]\right)_{i=1}^{N}$ is a sequence of admissible intervals

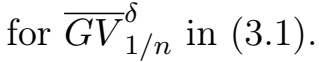

In all cases, when there is no admissible sequence $\left(\left[x_{i}, y_{i}\right]\right)_{i=1}^{N}$, we define the corresponding variation to be zero.

If $A \subset K \subset[a, b]$ are closed sets and $r G V_{1 / n}^{\delta}(f, A, K)<\infty$ (respectively, $\left.l G V_{1 / n}^{\delta}(f, A, K)<\infty\right)$, and $x \in A$, then $r G V_{1 / n}^{\delta}$ (resp. $\left.l G V_{1 / n}^{\delta}\right)$ is "additive at $x "$, i.e.

$$
\begin{aligned}
& r G V_{1 / n}^{\delta}(f, A, K) \\
& =r G V_{n}^{\delta}(f, A \cap[a, x], K \cap[a, x])+r G V_{n}^{\delta}(f, A \cap[x, b], K \cap[x, b])
\end{aligned}
$$

(and similarly for $l G V_{n}^{\delta}$ ). This is easily seen from the definition. Also,

$$
\begin{aligned}
\max & \left(l G V_{1 / n}^{\delta}(f, A, K), r G V_{1 / n}^{\delta}(f, A, K)\right) \\
& \leq \min \left(\overline{G V_{1 / n}^{\delta}}(f, A, K), G V_{1 / n}(f, A \cup\{a, b\}, K \cup\{a, b\})\right) .
\end{aligned}
$$

Further, if $0<\delta<\gamma$, then $\overline{G V}_{1 / n}^{\delta}(f, A, K) \leq \overline{G V}_{1 / n}^{\gamma}(f, A, K)$.

We will need some properties of the "unilateral" variations.

Lemma 3.2. Let $\emptyset \neq A \subset K \subset[a, b]$ be closed sets with $\{a, b\} \subset K$, and $f:[a, b] \rightarrow \mathbb{R}$ continuous and such that $\overline{G V}_{1 / n}^{\delta}(f, A, K)<\infty$ for some $\delta>0$ and $n \geq 2$. Then $v(x):=r G V_{1 / n}^{\delta}(f, A \cap[a, x], K \cap[a, x])$ and $\tilde{v}(x):=$ $l G V_{1 / n}^{\delta}(f, A \cap[x, b], K \cap[x, b])$ are continuous functions on $K$ such that $v$ is increasing, $\tilde{v}$ is decreasing, and

$$
\max (v(b)-v(a), \tilde{v}(a)-\tilde{v}(b)) \leq \overline{G V}_{1 / n}^{\delta}(f, A, K) .
$$

Proof. Clearly, $v$ is increasing and $\tilde{v}$ is decreasing on $K$. Also, we easily see that (3.4) holds. We will only establish the continuity of $v$ on $K$, as the case of $\tilde{v}$ is similar. Suppose that $x \in K$ is not a left-hand accumulation point of $A$. If $[x-\delta, x) \cap A=\emptyset$, then $v$ is constant on $[x-\delta, x]$. We will show that if $[x-\delta, x) \cap A \neq \emptyset$, then

$$
v(t)=v(r)+\left(V_{1 /(n-1)}(f, K \cap[r, t])\right)^{(n-1) / n}
$$


for all $t \in[r, x]$, where $r:=\max ([a, x) \cap A)$ provided $[a, x) \cap A \neq \emptyset$ (the case when $[a, x) \cap A=\emptyset$ is trivial). The $\geq$ inequality in (3.5) is obvious. The other inequality follows easily from the definition of the fractional variation together with the fact that $[x-\delta, x) \cap A=\emptyset$. The unilateral continuity of $v$ at $x$ in this case follows from [11, Lemma 3.13]. We have shown that $v$ is continuous from the left at all $x \in K$ which are not left-hand accumulation points of $A$.

If $x \in K$ is not a right-hand accumulation point of $A$, then either $(x-$ $\delta, x] \cap A=\emptyset$, in which case $v$ is constant on $[x, x+\eta]$ for some $\eta>0$, or $(x-\delta, x] \cap A \neq \emptyset$, and then (3.5) holds and the continuity of $v$ at $x$ from the right follows again from [11, Lemma 3.13].

Now suppose that $x \in A$ is a left-hand accumulation point of $A$. Fix $\varepsilon>0$. Choose $\left(\left[x_{i}, y_{i}\right]\right)_{i=1}^{N}$ as in (3.1) for $r G V_{1 / n}^{\delta}(f, A \cap[a, x], K \cap[a, x])$ and $\left(\left[c_{j}^{i}, d_{j}^{i}\right]\right)_{j=1}^{J_{i}}$ from $(2.1)$ for $V_{1 /(n-1)}\left(f,\left[x_{i}, y_{i}\right] \cap K\right)$ so that

$$
\sum_{i=1}^{N}\left(\sum_{j=1}^{J_{i}}\left|f\left(c_{j}^{i}\right)-f\left(d_{j}^{i}\right)\right|^{1 /(n-1)}\right)^{(n-1) / n}>v(x)-\varepsilon .
$$

We can assume that $\left(\left[x_{i}, y_{i}\right]\right)$ and $\left(\left[c_{j}^{i}, d_{j}^{i}\right]\right)$ are ordered in the natural sense (i.e. $y_{i}<x_{i^{\prime}}$ whenever $i<i^{\prime}$; similarly for $c_{j}^{i}$, $d_{j}^{i}$ ). We can suppose that $d_{J_{i}}^{N}<x$, because if $d_{J_{i}}^{N}=x$, then by continuity of $f$ and since $x$ is a left-hand accumulation point of $A$, we can make $d_{J_{i}}^{N}$ slightly smaller (and adjust $y_{N}$ ) without violating (3.6). Then we have $v(z)>v(x)-\varepsilon$ for every $d_{J_{i}}^{N}<z<x$ and hence $v$ is continuous from the left at $x$.

Now suppose that $x \in A$ is a a right-hand accumulation point of $A$, and fix $\varepsilon>0$. Choose $\left(\left[x_{i}, y_{i}\right]\right)_{i=1}^{N}$ and $\left(\left[c_{j}^{i}, d_{j}^{i}\right]\right)_{j=1}^{J_{i}}$ from (3.1) for $r G V_{1 / n}^{\delta}(f, A \cap$ $[x, b])=v(b)-v(x)$ (the equality holds because $x \in A$ ) so that

$$
\sum_{i=1}^{N}\left(\sum_{j=1}^{J_{i}}\left|f\left(c_{j}^{i}\right)-f\left(d_{j}^{i}\right)\right|^{1 /(n-1)}\right)^{(n-1) / n}>v(b)-v(x)-\varepsilon .
$$

As before, we can assume that $\left(\left[x_{i}, y_{i}\right]\right)$ and $\left(\left[c_{j}^{i}, d_{j}^{i}\right]\right)$ are ordered in the natural sense (see the remark after (3.6)) and that $c_{1}^{1}>x$ (with $x_{1}>x$ ). Let $y \in\left(x, x_{1}\right) \cap K$. Take any $\left(\left[\tilde{x}_{i}, \tilde{y}_{i}\right]\right)_{i=1}^{\tilde{N}}$ as in (3.1) for $r G V_{n}^{\delta}(f, A \cap[a, y])=v(y)$. We have to prove that

$$
\sum_{i=1}^{\tilde{N}}\left(V_{1 /(n-1)}\left(f,\left[\tilde{x}_{i}, \tilde{y}_{i}\right] \cap K\right)\right)^{(n-1) / n} \leq v(x)+\varepsilon .
$$

For $i=1, \ldots, \tilde{N}$ take $\left(\left[\tilde{c}_{j}^{i}, \tilde{d}_{j}^{i}\right]\right]_{j=1}^{\tilde{J}_{i}}$ as in $(2.1)$ for $V_{1 /(n-1)}\left(f,\left[\tilde{x}_{i}, \tilde{y}_{i}\right] \cap K\right)$. 
Since $a \leq \tilde{x}_{1}<\cdots<\tilde{x}_{N} \leq x_{1}<\cdots<x_{N} \leq b$, we have

$$
\begin{aligned}
\sum_{i=1}^{\tilde{N}}\left(\sum_{j=1}^{\tilde{J}_{i}} \mid f\left(\tilde{c}_{j}^{i}\right)\right. & \left.-\left.f\left(\tilde{d}_{j}^{i}\right)\right|^{1 /(n-1)}\right)^{(n-1) / n} \\
& +\sum_{i=1}^{N}\left(\sum_{j=1}^{J_{i}}\left|f\left(c_{j}^{i}\right)-f\left(d_{j}^{i}\right)\right|^{1 /(n-1)}\right)^{(n-1) / n} \leq v(b) .
\end{aligned}
$$

The left-hand side of (3.9) is by (3.7) greater than

$$
\sum_{i=1}^{\tilde{N}}\left(\sum_{j=1}^{\tilde{J}_{i}}\left|f\left(\tilde{c}_{j}^{i}\right)-f\left(\tilde{d}_{j}^{i}\right)\right|^{1 /(n-1)}\right)^{(n-1) / n}+v(b)-v(x)-\varepsilon,
$$

and this easily implies (3.8). This concludes the proof of the continuity of $v$ on $K$ since it is clearly an increasing function.

Now we are ready to define our classes of functions. The following class plays a similar rôle for $n$-times differentiability as the class $C B V_{1 / n}$ from [11] in the case of continuous derivatives.

Definition 3.3. We say that a continuous $f:[a, b] \rightarrow \mathbb{R}$ is $C B V G_{1 / n}$ for $n \geq 2$ provided $V_{1 /(n-1)}\left(f, K_{f}\right)<\infty$ and there exist closed $A_{m} \subset K_{f}$ $(m \in \mathcal{M} \subset \mathbb{N})$ such that $K_{f}=\bigcup_{m \in \mathcal{M}} A_{m}$, and $G V_{1 / n}\left(f, A_{m}, K_{f}\right)<\infty$ for all $m \in \mathcal{M}$.

It is easy to see that if $f$ is a $C B V G_{1 / n}$ function for some $n \geq 2$, then $f$ has bounded variation. If $n=2$, then it is not difficult to prove that the class $C B V G_{1 / 2}$ coincides with the class $V B G_{1 / 2}$ from [5].

The analogue of the class $S B V_{1 / n}$ from [11] in the case of continuous derivatives is given by the following definition for the case of $n$-times differentiable functions.

Definition 3.4. We say that a continuous $f:[a, b] \rightarrow \mathbb{R}$ is $S B V G_{1 / n}$ for $n \geq 2$ provided $V_{1 /(n-1)}\left(f, K_{f}\right)<\infty$ and there exist closed sets $A_{m} \subset K_{f}$ and numbers $\delta_{m}>0(m \in \mathbb{N})$ such that $K_{f}=\bigcup_{m \in \mathbb{N}} A_{m}$ and

(i) $\lim _{m \rightarrow \infty} \delta_{m}=0$,

(ii) $A_{m} \subset A_{m+1}$ for each $m \in \mathbb{N}$,

(iii) $\lim _{m \rightarrow \infty} \overline{G V}_{1 / n}^{\delta_{m}}\left(f, A_{m}, K_{f}\right)=0$.

We will need the following auxiliary class:

Definition 3.5. We say that a continuous $f:[a, b] \rightarrow \mathbb{R}$ is $\overline{S B V G}_{1 / n}$ for $n \geq 2$ provided $V_{1 /(n-1)}\left(f, K_{f}\right)<\infty$ and there exist closed sets $A_{m}^{k} \subset K_{f}$ and numbers $\delta_{m}^{k}>0(k, m \in \mathbb{N})$ such that $K_{f}=\bigcup_{k, m} A_{m}^{k}$ and for each $k \in \mathbb{N}$ we have 
(i) $\lim _{m \rightarrow \infty} \delta_{m}^{k}=0$,

(ii) $A_{m}^{k} \subset A_{m+1}^{k}$ for each $m \in \mathbb{N}$,

(iii) $\lim _{m \rightarrow \infty} \overline{G V}_{1 / n}^{\delta_{m}^{k}}\left(f, A_{m}^{k}, K_{f}\right)=0$.

Since every continuous function on a compact interval is uniformly continuous, it is easy to see that if $f$ is $S B V G_{1 / n}, \overline{S B V G}_{1 / n}$ or $C B V G_{1 / n}$ and $g$ is Lebesgue equivalent to $f$, then $g$ is in the same class.

We need the following observation:

Lemma 3.6. Let $f:[a, b] \rightarrow \mathbb{R}$. Then $f$ is $S B V G_{1 / n}$ if and only if $f$ is $\overline{S B V G}_{1 / n}$.

Proof. Suppose that $f$ is $S B V G_{1 / n}$. Let $A_{m}$ and $\delta_{m}(m \in \mathbb{N})$ be as in Definition 3.4. We define $A_{m}^{1}:=A_{m}$ and $A_{m}^{k}:=\emptyset$ for $k>1$. Similarly, $\delta_{m}^{1}:=\delta_{m}$ and $\delta_{m}^{k}:=1 / m$ for $k>1$. Then it is easy to see that $\left(A_{m}^{k}\right)$ and $\left(\delta_{m}^{k}\right)$ are as in Definition 3.5.

Now, suppose that $f$ is $\overline{S B V G}_{1 / n}$, and let $A_{m}^{k}$ and $\delta_{m}^{k}$ be as in Definition 3.5. By relabelling, we can assume that $A_{m}^{k} \neq \emptyset$ for all $m, k \in \mathbb{N}$ (since the case when there exists $k_{0} \in \mathbb{N}$ such that $\bigcup_{m} A_{m}^{k}=\emptyset$ for all $k \geq k_{0}$ is simple to handle using (3.10)). By a standard diagonalization argument (us-

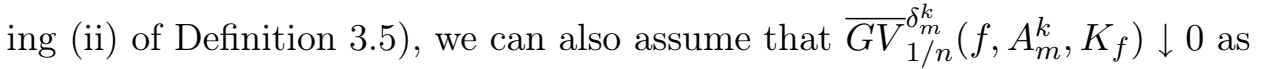
$m \rightarrow \infty$ for all $k \in \mathbb{N}$. Define $A_{1}:=A_{1}^{1}, \delta_{1}:=\delta_{1}^{1}$, and $N_{1}:=1$. By induction, we will construct closed sets $A_{p} \subset K_{f}, \delta_{p}>0$, and $N_{p} \in \mathbb{N}$. Suppose that $A_{1}, \ldots, A_{p-1}$ (together with $\delta_{i}$ and $N_{i}$ for $i<p$ ) have been constructed. Note that for closed $B_{1}, \ldots, B_{l} \subset K_{f}$, and $\xi>0$, we have

$$
\overline{G V}_{1 / n}^{\xi}\left(f, \bigcup_{j=1}^{l} B_{j}, K_{f}\right) \leq \sum_{j=1}^{l} \overline{G V_{1 / n}^{\xi}}\left(f, B_{j}, K_{f}\right) .
$$

For $i=1, \ldots, p$, find $l_{i}>N_{p-1}$ such that $\overline{G V}{ }^{\delta_{l_{i}}^{i}}\left(f, A_{l_{i}}^{i}, K_{f}\right) \leq p^{-2}$, put $A_{p}:=\bigcup_{i=1}^{p} A_{l_{i}}^{i}, \delta_{p}:=\min \left(p^{-1}, \min _{i=1, \ldots, p} \delta_{l_{i}}^{i}\right)$, and using (3.10) conclude that

$$
\overline{G V}_{1 / n}^{\delta_{p}}\left(f, A_{p}, K_{f}\right) \leq p^{-1} .
$$

Finally, put $N_{p}:=1+\max _{i=1, \ldots, p} l_{i}$, and proceed by induction. Since $K_{f}=$ $\bigcup_{k, m} A_{m}^{k}$, we obtain $K_{f}=\bigcup_{p} A_{p}$. By construction, it is easy to see that $A_{p} \subset A_{p+1}$ and $\delta_{p} \rightarrow 0$. Finally, (3.11) shows that

$$
\lim _{p \rightarrow \infty} \overline{G V}_{1 / n}^{\delta_{p}}\left(f, A_{p}, K_{f}\right)=0 .
$$

We will also need the following property.

Lemma 3.7. Let $f:[a, b] \rightarrow \mathbb{R}$ be a function which is either $C B V G_{1 / n}$, $\overline{S B V G}_{1 / n}$, or $S B V G_{1 / n}$. Then $\lambda\left(f\left(K_{f}\right)\right)=0$. 
Proof. If $f$ is in one of the three classes, then $V_{1 /(n-1)}\left(f, K_{f}\right)<\infty$, and thus Lemma 2.4 implies the conclusion.

The next lemma contains our basic estimate.

Lemma 3.8. Let $n \geq 2$, let $f:[a, b] \rightarrow \mathbb{R}$ be $(n-1)$-times differentiable with $f^{(n-1)}(\cdot)$ pointwise Lipschitz, let $K \subset\left\{x \in[a, b]: f^{\prime}(x)=0\right\}$ be closed with $a, b \in K$ and $|f(c)-f(d)|=V(f,[c, d])$ for all intervals $(c, d)$ contiguous to $K$ in $[a, b]$, let

$$
A \subset\left\{x \in K^{\prime}:\left|f^{(n-1)}(y)\right| \leq k|y-x| \text { for all } y \in B(x, 1 / m)\right\}
$$

be a closed set, where $k, m \in \mathbb{N}$, and let $x, x^{\prime} \in K$ be such that $a \leq x<x^{\prime} \leq b$, $\left\{x, x^{\prime}\right\} \cap A \neq \emptyset$ and $0<x^{\prime}-x<1 / m$. Then

$$
\begin{aligned}
\left(V_{1 /(n-1)}\left(f, K \cap\left[x, x^{\prime}\right]\right)\right)^{(n-1) / n} & \\
\leq & C_{k n}\left(x^{\prime}-x\right)^{1 / n}\left(\sum_{p \in \mathcal{P}}\left(v_{p}-u_{p}\right)\right)^{(n-1) / n},
\end{aligned}
$$

where $\left(u_{p}, v_{p}\right)(p \in \mathcal{P} \subset \mathbb{N})$ are all the intervals contiguous to $K$ in $\left[x, x^{\prime}\right]$, and $C_{k n}=k^{1 / n}(2 n)^{(n-1) / n}$.

Proof. Without any loss of generality, assume that $x \in A$ (if $x^{\prime} \in A$, then work with $f(-\cdot)$ instead). By Lemma 2.10 we have

$$
f^{(i)}(x)=0 \quad \text { for all } i=1, \ldots, n-1 .
$$

Let

$$
\begin{aligned}
& \left(\left[c_{j}, d_{j}\right]\right)_{j=1}^{J} \text { be nonoverlapping intervals } \\
& \text { with } c_{j}, d_{j} \in K \cap\left[x, x^{\prime}\right] \text { for all } j \in\{1, \ldots, J\} .
\end{aligned}
$$

Assume first that $\#\left(K \cap\left[x, x^{\prime}\right]\right)<2 n+1$. Then

$$
\begin{aligned}
\left|f\left(c_{j}\right)-f\left(d_{j}\right)\right| & \leq{ }_{c_{j}}^{d_{j}}\left|f^{\prime}(s)\right| d s \leq{ }_{c_{j}}^{d_{j}}\left|f^{(n-1)}\left(\xi_{n-1}\right)\right|\left(x^{\prime}-x\right)^{n-2} d s \\
& \leq k\left(x^{\prime}-x\right)^{n},
\end{aligned}
$$

where $\xi_{i}=\xi_{i}(s)$ is chosen inductively (using (3.13)) such that $\xi_{1}=s$, and

$$
\left|f^{(i-1)}\left(\xi_{i-1}\right)\right|=\left|f^{(i-1)}\left(\xi_{i-1}\right)-f^{(i-1)}(x)\right|=\left|f^{(i)}\left(\xi_{i}\right)\right|\left|\xi_{i-1}-x\right|
$$

for $i=2, \ldots, n-1$. We obtain $\left|f\left(c_{j}\right)-f\left(d_{j}\right)\right|^{1 /(n-1)} \leq k^{1 /(n-1)}\left(x^{\prime}-x\right)^{n /(n-1)}$, and it follows that

$$
\begin{aligned}
\sum_{j=1}^{J}\left|f\left(c_{j}\right)-f\left(d_{j}\right)\right|^{1 /(n-1)} & \leq k^{1 /(n-1)} \sum_{j=1}^{J}\left(x^{\prime}-x\right)^{n /(n-1)} \\
& \leq k^{1 /(n-1)}\left(x^{\prime}-x\right)^{1 /(n-1)}(2 n)\left(\sum_{p \in \mathcal{P}}\left(v_{p}-u_{p}\right)\right),
\end{aligned}
$$


where we used that $x^{\prime}-x=\sum_{p \in \mathcal{P}}\left(v_{p}-u_{p}\right)$ and $J \leq 2 n$ since $\#\left(K \cap\left[x, x^{\prime}\right]\right)<$ $2 n+1$. Thus

$$
\begin{aligned}
& \left(\sum_{j=1}^{J}\left|f\left(c_{j}\right)-f\left(d_{j}\right)\right|^{1 /(n-1)}\right)^{(n-1) / n} \\
& \quad \leq k^{1 / n}(2 n)^{(n-1) / n}\left(x^{\prime}-x\right)^{1 / n}\left(\sum_{p \in \mathcal{P}}\left(v_{p}-u_{p}\right)\right)^{(n-1) / n}
\end{aligned}
$$

and (3.12) holds in this case.

Now assume that $\#\left(K \cap\left[x, x^{\prime}\right]\right) \geq 2 n+1$. Let $\left(\left[c_{j}, d_{j}\right]\right)_{j=1}^{J}$ be as in (3.14). Since $\lambda(f(K))=0$ by Lemma 2.1, Lemma 2.5 implies that

$$
\begin{aligned}
\left|f\left(d_{j}\right)-f\left(c_{j}\right)\right| & \leq V\left(f,\left[c_{j}, d_{j}\right]\right)=\sum_{p \in \mathcal{P}_{j}} V\left(f,\left[\gamma_{p}^{j}, \delta_{p}^{j}\right]\right) \\
& =\sum_{p \in \mathcal{P}_{j}}\left|f\left(\delta_{p}^{j}\right)-f\left(\gamma_{p}^{j}\right)\right|,
\end{aligned}
$$

where we used the assumptions in the last equality, and where $\left(\gamma_{p}^{j}, \delta_{p}^{j}\right)$ $\left(p \in \mathcal{P}_{j} \subset \mathbb{N}\right)$ are all the intervals contiguous to $K \cap\left[c_{j}, d_{j}\right]$ in $\left[c_{j}, d_{j}\right]$. By adding (3.15) for $j=1, \ldots, J$, and using the subadditivity of $g(t)=t^{1 /(n-1)}$ for $t \geq 0$, we obtain

$$
\sum_{j=1}^{J}\left|f\left(c_{j}\right)-f\left(d_{j}\right)\right|^{1 /(n-1)} \leq \sum_{p \in \mathcal{P}}\left|f\left(v_{p}\right)-f\left(u_{p}\right)\right|^{1 /(n-1)} ;
$$

thus the conclusion of the lemma will follow once we establish

$$
\begin{aligned}
& \left(\sum_{p \in \mathcal{P}}\left|f\left(v_{p}\right)-f\left(u_{p}\right)\right|^{1 /(n-1)}\right)^{(n-1) / n} \\
& \quad \leq C_{k n}\left(x^{\prime}-x\right)^{1 / n}\left(\sum_{p \in \mathcal{P}}\left(v_{p}-u_{p}\right)\right)^{(n-1) / n} .
\end{aligned}
$$

To prove (3.17), we need the following definition. If $(\alpha, \beta)$ is an interval contiguous to $K \cap\left[x, x^{\prime}\right]$ in $\left[x, x^{\prime}\right]$, then put $r_{1}(\beta):=\beta$, and

$$
r_{i}(\beta):=\inf \left\{t \in\left[r_{i-1}(\beta), x^{\prime}\right]: \#(K \cap[\beta, t]) \geq i \text { or } t=x^{\prime}\right\}
$$

for $i=2, \ldots, n-2$. Similarly, define $l_{1}(\alpha):=\alpha$, and

$$
l_{i}(\alpha):=\sup \left\{t \in\left[x, l_{i-1}(\alpha)\right]: \#(K \cap[t, \alpha]) \geq i \text { or } t=x\right\}
$$

for $i=2, \ldots, n-2$. We will prove the following:

(*) for each interval $(\alpha, \beta)$ contiguous to $K \cap\left[x, x^{\prime}\right]$ in $\left[x, x^{\prime}\right]$ and each $i \in\{1, \ldots, n-2\}$ there exists $w_{i} \in\left[l_{i}(\alpha), r_{i}(\beta)\right]$ such that $f^{(i)}\left(w_{i}\right)=0$. 
If $i=1$, then take $w_{1}=\alpha$. Suppose that $1<i<n-1$. If $\left[l_{i}(\alpha), r_{i}(\beta)\right] \cap K^{\prime}$ $\neq \emptyset$, then $(*)$ follows by Lemma 2.10 ; otherwise $\#\left(\left[l_{i}(\alpha), r_{i}(\beta)\right] \cap K\right) \geq i+1$ by the choice of $l_{i}(\alpha), r_{i}(\beta)$, and $(*)$ follows from Lemma 2.9 .

Fix $p \in \mathcal{P}$ and let $s \in\left[u_{p}, v_{p}\right]$. Then $\left|f^{\prime}(s)\right|=\left|f^{\prime}(s)-f^{\prime}\left(u_{p}\right)\right|=\left|f^{\prime \prime}\left(\xi_{1}\right)\right|$. $\left|s-u_{p}\right|$, and by induction for $k=2, \ldots, n-2$, choose $\xi_{k} \in\left[l_{k}\left(u_{p}\right), r_{k}\left(v_{p}\right)\right]$ so that

$$
\left|f^{(k)}\left(\xi_{k-1}\right)\right|=\left|f^{(k)}\left(\xi_{k-1}\right)-f^{(k)}\left(w_{k}\right)\right|=\left|f^{(k+1)}\left(\xi_{k}\right)\right| \cdot\left|\xi_{k-1}-w_{k}\right|,
$$

where $w_{k}$ is chosen by applying $(*)$ to $\left(u_{p}, v_{p}\right)$. Using $(3.18)$, we obtain

$$
\left|f^{\prime}(s)\right| \leq\left|f^{(n-1)}\left(\xi_{n-2}\right)\right| \prod_{l=1}^{n-2}\left(r_{l}\left(v_{p}\right)-l_{l}\left(u_{p}\right)\right) .
$$

Thus we obtain

$$
\begin{aligned}
\left|f\left(u_{p}\right)-f\left(v_{p}\right)\right| & \leq{ }_{u_{p}}^{v_{p}}\left|f^{\prime}(s)\right| d s \leq{ }_{u_{p}}^{v_{p}}\left|f^{(n-1)}\left(\xi_{n-2}\right)\right| \prod_{l=1}^{n-2}\left(r_{l}\left(v_{p}\right)-l_{l}\left(u_{p}\right)\right) d s \\
& \leq k\left(v_{p}-x\right)\left(r_{n-2}\left(v_{p}\right)-l_{n-2}\left(u_{p}\right)\right)^{n-2}\left(v_{p}-u_{p}\right) .
\end{aligned}
$$

Hence $\left|f\left(u_{p}\right)-f\left(v_{p}\right)\right|^{1 /(n-1)} \leq\left(k\left(v_{p}-x\right)\right)^{1 /(n-1)}\left(r_{n-2}\left(v_{p}\right)-l_{n-2}\left(u_{p}\right)\right)$ for each $p \in \mathcal{P}$, and thus

$$
\begin{aligned}
\left(\sum_{p \in \mathcal{P}} \mid f\left(u_{p}\right)-\right. & \left.\left.f\left(v_{p}\right)\right|^{1 /(n-1)}\right)^{(n-1) / n} \\
& \leq k^{1 / n}\left(x^{\prime}-x\right)^{1 / n}\left(\sum_{p \in \mathcal{P}}\left(r_{n-2}\left(v_{p}\right)-l_{n-2}\left(u_{p}\right)\right)\right)^{(n-1) / n} .
\end{aligned}
$$

As $\sum_{p \in \mathcal{P}}\left(r_{n-2}\left(v_{p}\right)-l_{n-2}\left(u_{p}\right)\right) \leq 2 n \sum_{p \in \mathcal{P}}\left(v_{p}-u_{p}\right)$, we obtain

$$
\begin{aligned}
& \left(\sum_{p \in \mathcal{P}}\left|f\left(v_{p}\right)-f\left(u_{p}\right)\right|^{1 /(n-1)}\right)^{(n-1) / n} \\
& \quad \leq k^{1 / n}(2 n)^{(n-1) / n}\left(x^{\prime}-x\right)^{1 / n}\left(\sum_{p \in \mathcal{P}}\left(v_{p}-u_{p}\right)\right)^{(n-1) / n} .
\end{aligned}
$$

Thus, (3.17) and also (3.12) follow.

The following lemma contains a sufficient condition for a function to belong to the classes $\overline{S B V G}_{1 / n}$ and $C B V G_{1 / n}$.

Lemma 3.9. Suppose that $f:[a, b] \rightarrow \mathbb{R}$ is $(n-1)$-times differentiable $($ for $n \geq 2)$ so that $f^{(n-1)}(\cdot)$ is pointwise Lipschitz. Then $f$ is both $\overline{S B V G}_{1 / n}$ and $C B V G_{1 / n}$.

Proof. Note that $V_{1 /(n-1)}\left(f, K_{f}\right)<\infty$ by [11, Remark 3.6] since $f$ is $C^{n-1}$ by assumption. Since $K_{f} \subset\left\{x \in[a, b]: f^{\prime}(x)=0\right\}$, we have $f^{(n-1)}(x)$ 
$=0$ for $x \in K_{f}^{\prime}$ by Lemma 2.10. Denote

$$
B_{m}^{k}:=\left\{x \in K_{f}^{\prime}:\left|f^{(n-1)}(y)\right| \leq k|y-x| \text { for all } y \in B(x, 1 / m)\right\} .
$$

It is easy to see that each $B_{m}^{k}$ is closed, $\bigcup_{k, m} B_{m}^{k}=K_{f}^{\prime}$, and $B_{m}^{k} \subset B_{m+1}^{k}$. Let $\delta_{m}^{k}:=1 / 2 m$.

First, we will show that $\lim _{m \rightarrow \infty} \overline{G V}_{1 / n}^{\delta_{m}^{k}}\left(f, B_{m}^{k}, K_{f}\right)=0$. Fix $k, m \in \mathbb{N}$ with $B_{m}^{k} \neq \emptyset$. Let $\left(\left[x_{i}, y_{i}\right]\right)_{i=1}^{N}$ be as in (3.1) for $A=B_{m}^{k}, K=K_{f}$ and $\delta=\delta_{m}^{k}$

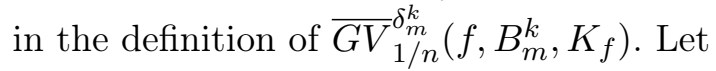

$$
\mathcal{P}_{i}:=\left\{p \in \mathcal{P}:\left(u_{p}, v_{p}\right) \subset\left[x_{i}, y_{i}\right]\right\},
$$

where $\left(u_{p}, v_{p}\right)(p \in \mathcal{P} \subset \mathbb{N})$ are all the intervals contiguous to $K_{f}$ in $[a, b]$. Applying Lemma 3.8 to $\left[x, x^{\prime}\right]=\left[x_{i}, y_{i}\right]$ for a fixed $i=1, \ldots, N$, summing over $i \in\{1, \ldots, N\}$, and using Hölder's inequality (with exponents $p=n$ and $\left.p^{\prime}=n /(n-1)\right)$, we obtain

$$
\begin{aligned}
\sum_{i=1}^{N}\left(V_{1 /(n-1)}(f,\right. & \left.\left.K_{f} \cap\left[x_{i}, y_{i}\right]\right)\right)^{(n-1) / n} \\
& \leq C_{k n} \sum_{i=1}^{N}\left(y_{i}-x_{i}\right)^{1 / n} \cdot\left(\sum_{p \in \mathcal{P}_{i}}\left(v_{p}-u_{p}\right)\right)^{(n-1) / n} \\
& \leq C_{k n}(b-a)^{1 / n}\left(\sum_{i=1}^{N} \sum_{p \in \mathcal{P}_{i}}\left(v_{p}-u_{p}\right)\right)^{(n-1) / n} \\
& \leq C_{k n}(b-a)^{1 / n}\left(\sum_{v_{p}-u_{p} \leq \delta_{m}^{k}}\left(v_{p}-u_{p}\right)\right)^{(n-1) / n}
\end{aligned}
$$

and since $\lim _{m \rightarrow \infty} \delta_{m}^{k}=0$ for a fixed $k \in \mathbb{N}$, by (3.20) we obtain

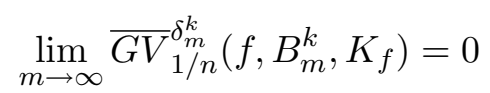

for each $k \in \mathbb{N}$.

Enumerate $K_{f} \backslash K_{f}^{\prime}$ as $\left\{x_{j}\right\}_{j \in \mathcal{J}}$, where $\mathcal{J} \subset \mathbb{N}$ and each $j \in \mathcal{J}$ is even. Set $A_{m}^{2 k+1}:=B_{m}^{2 k+1}$ and $\tilde{\delta}_{m}^{2 k+1}:=\delta_{m}^{2 k+1}$. For $k \in \mathcal{J}$ define $A_{m}^{k}:=\left\{x_{k}\right\}$ for all $m \in \mathbb{N}$. For each $k \in \mathcal{J}$ find $\gamma_{k}>0$ such that $B\left(x_{k}, \gamma_{k}\right) \backslash\left\{x_{k}\right\} \cap K_{f}=\emptyset$ and put $\tilde{\delta}_{m}^{k}:=\min \left(\gamma_{k}, 1 / m\right)$. For $k \in \mathbb{N} \backslash \mathcal{J}$ even, put $A_{m}^{k}:=\emptyset$ for all $m \in \mathbb{N}$ and $\tilde{\delta}_{m}^{k}:=1 / m$. Using (3.21), it is easy to see that the conditions of Definition 3.5 are satisfied with $A_{m}^{k}$ and $\tilde{\delta}_{m}^{k}$.

To show that $f$ is $C B V G_{1 / n}$, write each $B_{m}^{k}$ as $B_{m}^{k}=\bigcup_{l} B_{m l}^{k}$, where each $B_{m l}^{k}$ is closed and $\operatorname{diam}\left(B_{m l}^{k}\right)<1 / m$. Fix $k, m, l \in \mathbb{N}$ such that $B_{m l}^{k} \neq \emptyset$. Let $\left(\left[x_{i}, y_{i}\right]\right)_{i=1}^{N}, x_{i}, y_{i} \in B_{m l}^{k}$, be as in (3.1) for $f, A=B_{m l}^{k}, K=K_{f}$ and the definition of $G V_{1 / n}\left(f, B_{m l}^{k}, K_{f}\right)$. If $i \in\{1, \ldots, N\}$, then Lemma 3.8 applied 
to $\left[x, x^{\prime}\right]=\left[x_{i}, y_{i}\right]$ shows that

$$
\begin{aligned}
& \left(V_{1 /(n-1)}\left(f,\left[x_{i}, y_{i}\right] \cap K_{f}\right)\right)^{(n-1) / n} \\
& \quad \leq C_{k n}\left(y_{i}-x_{i}\right)^{1 / n}\left(\sum_{i \in \mathcal{P}_{i}}\left(v_{p}-u_{p}\right)\right)^{(n-1) / n} \leq C_{k n}\left(y_{i}-x_{i}\right),
\end{aligned}
$$

where $\mathcal{P}_{i}$ is defined in (3.19). By summing over $i \in\{1, \ldots, N\}$ in (3.22), we obtain

$$
\begin{aligned}
\sum_{i=1}^{N}\left(V_{1 /(n-1)}\left(f,\left[x_{i}, y_{i}\right] \cap K_{f}\right)\right)^{(n-1) / n} & \leq C_{k n} \sum_{i=1}^{N}\left(y_{i}-x_{i}\right) \\
& \leq C_{k n}(b-a)<\infty .
\end{aligned}
$$

We have proved that $G V_{1 / n}\left(f, B_{m l}^{k}, K_{f}\right)<\infty$ (for each $\left.k, m, l \in \mathbb{N}\right)$. If we reorder the sequence $\left(B_{m l}^{k}\right)_{k, m, l}$ together with the sequence $(\{x\})_{x \in K_{f} \backslash K_{f}^{\prime}}$ into a single sequence which we call $A_{m}$ (omitting the empty sets), where $m \in \mathcal{M} \subset \mathbb{N}$, by (3.23) we see that $f$ is $C B V G_{1 / n}$.

The following lemma will allow us to construct certain variations which play a key rôle in establishing differentiability.

Lemma 3.10. Let $f:[a, b] \rightarrow \mathbb{R}$ be continuous, $\emptyset \neq A \subset K \subset[a, b]$ be

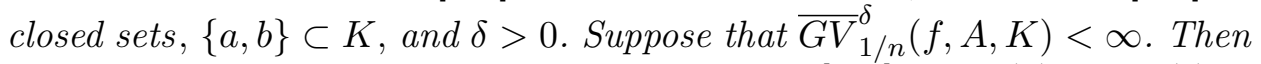
there exists a continuous increasing function $v$ on $[a, b]$ with $v(a)=0, v(b) \leq$ $\overline{G V_{1 / n}^{\delta}}(f, A)$, and such that for $x \in A$ and $y, z \in K$ with $x \leq y<z<x+\delta$ we have

$$
|f(y)-f(z)| \leq n^{n-1}(v(z)-v(y))^{n-1}(v(z)-v(x)) .
$$

If $f$ has bounded variation, and $V(f,[\alpha, \beta])=|f(\beta)-f(\alpha)|$ whenever $(\alpha, \beta)$ is an interval contiguous to $K$, then $\lambda(v(K))=0$.

Proof. Define $v(x):=r G V_{n}^{\delta}(f, A \cap[a, x], K \cap[a, x])$ for $x \in K$. The continuity of $v$ on $K$ follows from Lemma 3.2. Now, extend $v$ to a continuous function on $[a, b]$ such that $v$ is continuous and affine on $[\alpha, \beta]$ whenever $(\alpha, \beta)$ is an interval contiguous to $K$ in $[a, b]$.

To prove (3.24), let $x \leq y<z<x+\delta$ where $x \in A$ and $y, z \in K$. By continuity, there is no loss of generality in assuming that $x<y$. Fix $\varepsilon_{0}>0$. Choose $\left(\left[x_{i}, y_{i}\right]\right)_{i=1}^{N}$ (see (3.1)) and $\left(\left[c_{j}^{i}, d_{j}^{i}\right]\right]_{j=1}^{J_{i}}$ with $c_{j}^{i}, d_{j}^{i} \in K \cap\left[x_{i}, y_{i}\right]$ such that

$$
v(y)=v(x)+\sum_{i=1}^{N}\left(\sum_{j=1}^{J_{i}}\left|f\left(d_{j}^{i}\right)-f\left(c_{j}^{i}\right)\right|^{1 /(n-1)}\right)^{(n-1) / n}+\varepsilon,
$$

where $0 \leq \varepsilon<\varepsilon_{0}$. This can be done because $x \in A$ (see (3.2)). We can also assume that $\left(\left[x_{i}, y_{i}\right]\right)$ and $\left(\left[c_{j}^{i}, d_{j}^{i}\right]\right)$ are ordered in the natural sense (see the 
remark after (3.6)). Then

$$
\begin{aligned}
v(z) \geq & v(x)+\sum_{i=1}^{N-1}\left(\sum_{j=1}^{J_{i}}\left|f\left(d_{j}^{i}\right)-f\left(c_{j}^{i}\right)\right|^{1 /(n-1)}\right)^{(n-1) / n} \\
& +\left(\sum_{j=1}^{J_{N}}\left|f\left(d_{j}^{N}\right)-f\left(c_{j}^{N}\right)\right|^{1 /(n-1)}+|f(y)-f(z)|^{1 /(n-1)}\right)^{(n-1) / n} .
\end{aligned}
$$

To simplify the notation, put $b:=\sum_{j=1}^{J_{N}}\left|f\left(d_{j}^{N}\right)-f\left(c_{j}^{N}\right)\right|^{1 /(n-1)}$ and $a:=$ $b+|f(y)-f(z)|^{1 /(n-1)}$. Because of the algebraic identity

$$
u^{(n-1) / n}-w^{(n-1) / n}=(u-w) \cdot \frac{\sum_{i=0}^{n-2} u^{i / n} w^{(n-2-i) / n}}{\sum_{i=0}^{n-1} u^{i / n} w^{(n-1-i) / n}},
$$

which is easily seen to be valid for all $u, w \geq 0$ with $u+w>0$, we obtain

$$
v(z)-v(y) \geq a^{(n-1) / n}-b^{(n-1) / n}-\varepsilon=(a-b) \cdot \frac{\sum_{i=0}^{n-2} a^{i / n} b^{(n-2-i) / n}}{\sum_{i=0}^{n-1} a^{i / n} b^{(n-1-i) / n}}-\varepsilon .
$$

Because $a \geq b$, we obtain $\sum_{i=0}^{n-1} a^{i / n} b^{(n-1-i) / n} \leq n a^{(n-1) / n}$, and this together with the inequality $v(z)-v(x) \geq a^{(n-1) / n}$ implies

$$
\begin{aligned}
v(z)-v(y) & \geq|f(y)-f(z)|^{1 /(n-1)} \cdot \frac{a^{(n-2) / n}}{n a^{(n-1) / n}}-\varepsilon \\
& =\frac{|f(y)-f(z)|^{1 /(n-1)}}{n a^{1 / n}}-\varepsilon \geq \frac{|f(y)-f(z)|^{1 /(n-1)}}{n(v(z)-v(x))^{1 /(n-1)}}-\varepsilon .
\end{aligned}
$$

To finish the proof of $(3.24)$, let $\varepsilon_{0} \rightarrow 0$.

Now, suppose $f$ has bounded variation and $V(f,[\alpha, \beta])=|f(\beta)-f(\alpha)|$ whenever $(\alpha, \beta)$ is an interval contiguous to $K$. We will show that $\lambda(v(K))$ $=0$. Let $\left(c_{p}, d_{p}\right)(p \in \mathcal{P} \subset \mathbb{N})$ be all the intervals contiguous to $K$ in $[a, b]$. First, we will prove that

$$
v(b)-v(a) \leq \sum_{p \in \mathcal{P}}\left(v\left(d_{p}\right)-v\left(c_{p}\right)\right) .
$$

Fix $\varepsilon_{0}>0$, and let $\left(\left[x_{i}, y_{i}\right]\right)_{i=1}^{N}$ be nonoverlapping intervals as in Definition 3.1 for $r G V_{1 / n}^{\delta}(f, A, K)$ such that

$$
v(b)-v(a)=\sum_{i=1}^{N}\left(V_{1 /(n-1)}\left(f, K \cap\left[x_{i}, y_{i}\right]\right)\right)^{(n-1) / n}+\varepsilon,
$$

for some $0 \leq \varepsilon<\varepsilon_{0} / 3$. Now for each $i=1, \ldots, N$, find nonoverlapping intervals $\left(\left[c_{j}^{i}, d_{j}^{i}\right]\right)_{j=1}^{J_{i}}$ in $\left[x_{i}, y_{i}\right]$ such that $c_{j}^{i}, d_{j}^{i} \in K \cap\left[x_{i}, y_{i}\right]$ and

$$
V_{1 /(n-1)}\left(f, K \cap\left[x_{i}, y_{i}\right]\right) \leq \sum_{j=1}^{J_{i}}\left|f\left(d_{j}^{i}\right)-f\left(c_{j}^{i}\right)\right|^{1 /(n-1)}+\left(\frac{\varepsilon_{0}}{3 N}\right)^{n /(n-1)} .
$$


For $i \in\{1, \ldots, N\}$, we can assume that $d_{j}^{i} \leq c_{j+1}^{i}$ for $j=1, \ldots, J^{i}-1$. For the moment, fix $i \in\{1, \ldots, N\}$. By splitting and regrouping the intervals $\left(\left[c_{j}^{i}, d_{j}^{i}\right]\right)_{j=1}^{J_{i}}$, we can assume that there is a sequence of finite families of intervals $\mathcal{A}_{k}^{i}, k=1, \ldots, K_{i}$, and points $a_{k}^{i} \in A \cap\left[x_{i}, y_{i}\right]$ such that if $(\alpha, \beta) \in$ $\mathcal{A}_{k}^{i}$, then $\alpha, \beta \in K \cap\left[x_{i}, y_{i}\right]$, if $(\sigma, \tau) \in \mathcal{A}_{l}^{i}$ where $k<l$, then $\alpha<\beta \leq a_{l}^{i} \leq$ $\sigma<\tau$,

$$
\sum_{j=1}^{J_{i}}\left|f\left(d_{j}^{i}\right)-f\left(c_{j}^{i}\right)\right|^{1 /(n-1)} \leq \sum_{k=1}^{K_{i}} \sum_{(\alpha, \beta) \in \mathcal{A}_{k}^{i}}|f(\beta)-f(\alpha)|^{1 /(n-1)},
$$

and

$$
\left(c_{j}^{i}, d_{j}^{i}\right) \subset \overline{\bigcup\left\{(\alpha, \beta):(\alpha, \beta) \in \mathcal{A}_{k}^{i}, k=1, \ldots, K_{i}\right\}} .
$$

By Lemma 2.5 applied to $f$ on $[a, b]=[\alpha, \beta]$ for $(\alpha, \beta) \in \mathcal{A}_{k}^{i}$, and $B=$ $(A \cup\{\alpha, \beta\}) \cap[\alpha, \beta]$ (note that $\lambda(f(A))=0$ since $\lambda(f(K))=0$, and thus $\lambda(f(B))=0)$, let $\left(\alpha_{l}^{\alpha, \beta}, \beta_{l}^{\alpha, \beta}\right)\left(l \in\left\{1, \ldots, L_{k}^{i}\right\}\right)$ be a finite collection of intervals contiguous to $(A \cup\{\alpha, \beta\}) \cap[\alpha, \beta]$ in $[\alpha, \beta]$ such that

$$
|f(\beta)-f(\alpha)| \leq V(f,[\alpha, \beta]) \leq \sum_{l=1}^{L_{i k}} V\left(f,\left[\alpha_{l}^{\alpha, \beta}, \beta_{l}^{\alpha, \beta}\right]\right)+\left(\frac{\varepsilon_{0}}{3 N K_{i}\left|\mathcal{A}_{k}^{i}\right|}\right)^{n} .
$$

On the set $\left\{\left(\alpha_{l}^{\alpha, \beta}, \beta_{l}^{\alpha, \beta}\right): l=1, \ldots, L_{i k}, k=1, \ldots, K_{i},(\alpha, \beta) \in \mathcal{A}_{k}^{i}\right\}$ define an equivalence relation $\sim$ in the following way: $I \sim J$ whenever $(\max I, \min J) \cap A=\emptyset$ and $(\max J, \min I) \cap A=\emptyset$ (note that one of the conditions always holds). Denote by $\mathcal{B}_{q}^{i}\left(q=1, \ldots, Q_{i}\right)$ the equivalence classes of $\sim$. We have

$$
\begin{aligned}
& \sum_{k=1}^{K_{i}} \sum_{(\alpha, \beta) \in \mathcal{A}_{k}^{i}}|f(\beta)-f(\alpha)|^{1 /(n-1)} \\
& \quad \leq \sum_{k=1}^{K_{i}} \sum_{(\alpha, \beta) \in \mathcal{A}_{k}^{i}} \sum_{l=1}^{L_{i k}}\left(V\left(f,\left[\alpha_{l}^{\alpha, \beta}, \beta_{l}^{\alpha, \beta}\right]\right)\right)^{1 /(n-1)}+\left(\frac{\varepsilon_{0}}{3 N}\right)^{n /(n-1)} \\
& \quad \leq \sum_{q=1}^{Q_{i}} \sum_{(\eta, \theta) \in \mathcal{B}_{q}^{i}}(V(f,[\eta, \theta]))^{1 /(n-1)}+\left(\frac{\varepsilon_{0}}{3 N}\right)^{n /(n-1)} .
\end{aligned}
$$

By Lemma 2.5 and the assumptions, we obtain

$$
\begin{aligned}
\sum_{(\eta, \theta) \in \mathcal{B}_{q}^{i}}(V(f,[\eta, \theta]))^{1 /(n-1)} & \leq \sum_{\xi \in \Xi_{q}^{i}}\left|f\left(\omega_{\xi}\right)-f\left(\Omega_{\xi}\right)\right|^{1 /(n-1)} \\
& \leq V_{1 /(n-1)}\left(f,\left[\tau_{q}^{i}, T_{q}^{i}\right]\right),
\end{aligned}
$$

where $\left(\omega_{\xi}, \Omega_{\xi}\right)$ (for $\xi \in \Xi_{q}^{i} \subset \mathbb{N}$ ) are all the intervals contiguous to $K \cap\left[\tau_{q}^{i}, T_{q}^{i}\right]$ 
for $\tau_{q}^{i}=\inf _{x \in I \in \mathcal{B}_{q}^{i}} x$ and $T_{q}^{i}=\sup _{x \in I \in \mathcal{B}_{q}^{i}} x$. By combining the inequalities (3.26)-(3.30), we obtain

$$
\begin{aligned}
v(b)-v(a) & \leq \sum_{i=1}^{N}\left(\sum_{j=1}^{J_{i}}\left|f\left(d_{j}^{i}\right)-f\left(c_{j}^{i}\right)\right|^{1 /(n-1)}\right)^{(n-1) / n}+\frac{2 \varepsilon_{0}}{3} \\
& \leq \sum_{i=1}^{N}\left(\sum_{k=1}^{K_{i}} \sum_{(\alpha, \beta) \in \mathcal{A}_{k}^{i}}|f(\beta)-f(\alpha)|^{1 /(n-1)}\right)^{(n-1) / n}+\frac{2 \varepsilon_{0}}{3} \\
& \leq \sum_{i=1}^{N} \sum_{q=1}^{Q_{i}}\left(\sum_{(\eta, \theta) \in \mathcal{B}_{q}^{i}}(V(f,[\eta, \theta]))^{1 /(n-1)}\right)^{(n-1) / n}+\varepsilon_{0} \\
& \leq \sum_{i=1}^{N} \sum_{q=1}^{Q_{i}}\left(V_{1 /(n-1)}\left(f,\left[\tau_{q}^{i}, T_{q}^{i}\right] \cap K\right)\right)^{(n-1) / n}+\varepsilon_{0} .
\end{aligned}
$$

Since $\left(\tau_{q}^{i}, T_{q}^{i}\right) \cap A=\emptyset$, for $x \in\left[\tau_{q}^{i}, T_{q}^{i}\right] \cap K$ we have

$$
v(x)=v(z)+\left(V_{1 /(n-1)}(f, K \cap[z, x])\right)^{(n-1) / n},
$$

where $z=\max \left(A \cap\left[a, \tau_{q}^{i}\right]\right)$, Lemma 2.7 shows that $\lambda\left(v\left(\left[\tau_{q}^{i}, T_{q}^{i}\right] \cap K\right)\right)=0$. Now, Lemma 2.5 applied to $\zeta(x)\left(\right.$ where $\zeta(x)=\left(V_{1 /(n-1)}(f, K \cap[z, x])\right)^{(n-1) / n}$ for $x \in\left[\tau_{q}^{i}, T_{q}^{i}\right] \cap K$, and $\zeta$ is continuous and affine on intervals contiguous to $\left.\left[\tau_{q}^{i}, T_{q}^{i}\right] \cap K\right)$ implies that

$$
\left(V_{1 /(n-1)}\left(f,\left[\tau_{q}^{i}, T_{q}^{i}\right] \cap K\right)\right)^{(n-1) / n}=v\left(T_{q}^{i}\right)-v\left(\tau_{q}^{i}\right) \leq \sum_{p \in \mathcal{P}_{q}^{i}}\left(v\left(d_{p}\right)-v\left(c_{p}\right)\right),
$$

where $\mathcal{P}_{q}^{i}=\left\{p \in \mathcal{P}:\left(c_{p}, d_{p}\right) \subset\left[\tau_{q}^{i}, T_{q}^{i}\right]\right\}$. Combining this inequality with (3.31), we get $v(b)-v(a) \leq \sum_{p \in \mathcal{P}}\left(v\left(d_{p}\right)-v\left(c_{p}\right)\right)+\varepsilon_{0}$, and by letting $\varepsilon_{0} \rightarrow 0$ it follows that (3.25) holds. Since $v(K) \cap v\left(\bigcup_{p \in \mathcal{P}}\left(c_{p}, d_{p}\right)\right)$ is countable, we have $\lambda(v(K))=v(b)-v(a)-\lambda\left(\bigcup_{p \in \mathcal{P}}\left(c_{p}, d_{p}\right)\right)=0$.

By a symmetric argument (this time defining $\left.\tilde{v}(x):=l G V_{n}^{\delta}(f, A \cap[x, b])\right)$, we obtain the following:

Lemma 3.11. Let $f:[a, b] \rightarrow \mathbb{R}$ be continuous, $\emptyset \neq A \subset K \subset[a, b]$ be closed sets, $\{a, b\} \subset K$, and $\delta>0$. Suppose that $\overline{G V}_{1 / n}^{\delta}(f, A, K)<\infty$. Then there exists a continuous decreasing function $\tilde{v}$ on $[a, b]$ with $\tilde{v}(b)=0$, $\tilde{v}(a) \leq \overline{G V}_{1 / n}^{\delta}(f, A, K)$, and such that for $x \in A$ and $y, z \in K$ with $x-\delta<$ $z<y \leq x$ we have

$$
|f(y)-f(z)| \leq n^{n-1}(\tilde{v}(z)-\tilde{v}(y))^{n-1}(\tilde{v}(z)-\tilde{v}(x)) .
$$

If $\{a, b\} \subset K, f$ has bounded variation, and $V(f,[\alpha, \beta])=|f(\beta)-f(\alpha)|$ whenever $(\alpha, \beta)$ is an interval contiguous to $K$, then $\lambda(\tilde{v}(K))=0$. 
We have the following proposition.

Proposition 3.12. Let $f:[a, b] \rightarrow \mathbb{R}$ be an $S B V G_{1 / n}$ function. Then $f$ is Lebesgue equivalent to an $n$-times differentiable function $\varphi$ such that $\varphi^{(i)}(x)=0$ whenever $i \in\{1, \ldots, n\}$ and $x \in K_{\varphi}$. Also, $\varphi^{\prime}(x) \neq 0$ whenever $x \in[a, b] \backslash K_{\varphi}$. If $f$ is not constant on any interval, then $\lambda\left(K_{\varphi}\right)=0$.

Proof. For the moment, assume that $f$ is not constant in any interval. Lemma 2.6 shows that $V(f,[a, b])<\infty$. Then put $g:=f \circ v_{f}^{-1}:[0, \ell] \rightarrow \mathbb{R}$ (where $\left.\ell:=v_{f}(b)\right)$. Since $\lambda\left(f\left(K_{f}\right)\right)=0$ from Lemma 2.4, and $v_{f}\left(K_{f}\right)=K_{g}$, from Lemma 2.5 we have

$$
\ell=V(f,[a, b])=\sum_{p \in \mathcal{P}} V\left(g,\left[u_{p}, v_{p}\right]\right)=\sum_{p \in \mathcal{P}}\left(v_{p}-u_{p}\right),
$$

where $\left(u_{p}, v_{p}\right)(p \in \mathcal{P} \subset \mathbb{N})$ are all the intervals contiguous to $K_{g}$ in $[0, \ell]$, and thus $\lambda\left(K_{g}\right)=\ell-\lambda\left(\bigcup_{p \in \mathcal{P}}\left(u_{p}, v_{p}\right)\right)=0$. Putting $G(t)=g\left(\frac{\ell}{b-a} \cdot(t-a)\right)$, $t \in[a, b]$, we can assume that $f$ satisfies $\lambda\left(K_{f}\right)=0$ (since $f$ is clearly Lebesgue equivalent to $G$ ) provided $f$ is not constant in any interval.

Let $\left(A_{m}\right)_{m}$ and $\left(\delta_{m}\right)_{m} \subset \mathbb{R}_{+} \backslash\{0\}$ be the sequences from Definition 3.4 for $f$. Find a monotone sequence $\left(m_{j}\right)_{j \in \mathbb{N}} \subset \mathbb{N}$ such that $\lim _{j \rightarrow \infty} m_{j}=\infty$, and

$$
\sum_{j} j \cdot \overline{G V}_{1 / n}^{\delta_{m_{j}}}\left(f, A_{m_{j}}, K_{f}\right)<\infty .
$$

Relabel $\left(A_{m_{j}}\right)_{j}$ as $\left(A_{m}\right)_{m}$, and $\left(\delta_{m_{j}}\right)_{j}$ as $\left(\delta_{m}\right)_{m}$. Then by (3.34) we have

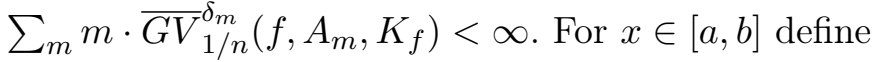

$$
v(x):=x+\sum_{m} m\left(v_{m}(x)-\tilde{v}_{m}(x)\right),
$$

where $v_{m}(x)$ (resp. $\tilde{v}_{m}(x)$ ) are the functions $v$ (resp. $\tilde{v}$ ) obtained by applying Lemma 3.10 (resp. Lemma 3.11) to $f, K=K_{f}, A=A_{m}$, and $\delta=\delta_{m}$. Note that $v: K_{f} \rightarrow[c, d]$ is a continuous strictly increasing function, which maps onto $[c, d]$, where $c=v(a), d=v(b)$.

If $f$ is not constant on any interval, by Lemmata 3.10 and 3.11, we have $\lambda\left(v_{m}\left(K_{f}\right)\right)=0$ and $\lambda\left(\tilde{v}_{m}\left(K_{f}\right)\right)=0$ for each $m \in \mathbb{N}$. Also, $\lambda\left(K_{f}\right)=0$, and thus Lemma 2.2 shows that

$$
\lambda\left(v\left(K_{f}\right)\right)=0 .
$$

For $x \in K_{f}$, we will show that for each $\varepsilon>0$ there exists $\delta>0$ such that if $x \leq y<z<x+\delta$ or $x-\delta<z<y \leq x$, and $y, z \in K_{f}$, then

$$
|f(y)-f(z)| \leq \varepsilon|v(z)-v(y)|^{n-1}|v(z)-v(x)| .
$$

Fix $x \in K_{f}$ and $\varepsilon>0$. Find $m_{0} \in \mathbb{N}$ such that $x \in A_{m_{0}}$ (and thus $x \in A_{m}$ for all $m \geq m_{0}$ ), and pick $m>m_{0}$ such that $n^{n-1} / m^{n}<\varepsilon$. Define $\delta:=\delta_{m}$. Let $y, z \in K_{f}$ be such that $x<y<z<x+\delta$. Then (3.24) implies that 
$|f(y)-f(z)| \leq n^{n-1}\left(v_{m}(z)-v_{m}(y)\right)^{n-1}\left(v_{m}(z)-v_{m}(x)\right)$. But since we have $m\left(v_{m}(\tau)-v_{m}(\sigma)\right) \leq v(\tau)-v(\sigma)$ for all $a \leq \sigma<\tau \leq b$, we obtain

$$
m^{n}|f(y)-f(z)| \leq n^{n-1}(v(z)-v(y))^{n-1}(v(z)-v(x)) .
$$

By the choice of $m$ we have $|f(y)-f(z)| \leq \varepsilon(v(z)-v(y))^{n-1}(v(z)-v(x))$. By continuity, the above argument shows that (3.36) also holds for $y, z \in K_{f}$ such that $x=y<z<x+\delta$. Finally, by using (3.33) (instead of (3.24)) in the previous argument, we obtain (3.36) for $x-\delta<z<y \leq x$ with $y, z \in K_{f}$.

We define $F:[c, d] \rightarrow \mathbb{R}$ as

$$
F(x):= \begin{cases}f \circ v^{-1}(x) & \text { for } x \in v\left(K_{f}\right), \\ H_{\alpha, \beta}(x) & \text { for } x \in(\alpha, \beta),\end{cases}
$$

whenever $(\alpha, \beta)$ is an interval contiguous to $v\left(K_{f}\right)$ in $[c, d]$, and $H=H_{\alpha, \beta}$ is chosen by applying Lemma 2.11 to $\alpha, \beta, A=f \circ v^{-1}(\alpha), B=f \circ v^{-1}(\beta)$. It follows that $F$ is Lebesgue equivalent to $f$, and $F$ is $n$-times differentiable at all $x \in[c, d] \backslash v\left(K_{f}\right)$ (by Lemma 2.11). To prove that $F$ is $n$-times differentiable, it remains to show that $F^{(i)}(x)=0$ for all $x \in v\left(K_{f}\right), i=1, \ldots, n$.

Now, (3.36) implies that for each $x \in v\left(K_{f}\right)$ and for each $\varepsilon>0$ there exists $\delta>0$ such that

$$
|F(y)-F(z)| \leq \varepsilon|y-z|^{n-1}|x-z|
$$

whenever $x \leq y<z<x+\delta$ or $x-\delta<z<y \leq x$, and $y, z \in v\left(K_{f}\right)$. Fix $x \in v\left(K_{f}\right)$. First, we will show that for each $x \in v\left(K_{f}\right)$ we have

$$
\text { for each } \varepsilon>0 \text { there exists } \delta>0 \text { such that }
$$

$$
\left|F^{(n-1)}(t)\right| \leq \varepsilon|t-x| \text { for all } t \in[c, d] \backslash v\left(K_{f}\right) \text { with }|x-t|<\delta .
$$

If $x$ is not a right-hand accumulation point of $v\left(K_{f}\right)$, then (3.39) follows from (3.37) and the fact that $H_{\alpha, \beta}^{(n)}(x)=0$ (where $x=\alpha$, and $(\alpha, \beta)$ is the corresponding interval contiguous to $v\left(K_{f}\right)$ ). If $x$ is a right-hand accumulation point of $v\left(K_{f}\right)$, let $\varepsilon>0$ be given, and choose $\delta>0$ such that (3.38) holds and $x+\delta \in v\left(K_{f}\right)$. Let $(\alpha, \beta)$ be an interval contiguous to $v\left(K_{f}\right)$ in $[c, d]$ with $(\alpha, \beta) \subset[x, x+\delta]$, and let $t \in(\alpha, \beta)$ (the case $t<x$ being symmetric). Let $l_{1}(x):=\xi(x-\alpha)$ for $x \in[\alpha,(\alpha+\beta) / 2]$, and $l_{2}(x):=\xi(\beta-x)$ for $x \in[(\alpha+\beta) / 2, \beta]$, where $\xi=C \cdot\left|f \circ v^{-1}(\beta)-f \circ v^{-1}(\alpha)\right| /(\beta-\alpha)^{n}$, and $C=C_{\alpha, \beta}$ comes from condition (iii) of Lemma 2.11 applied on $[\alpha, \beta]$. By (3.38), we have

$$
\begin{aligned}
l_{i}\left(\frac{\alpha+\beta}{2}\right) & =C \cdot \frac{\left|f \circ v^{-1}(\beta)-f \circ v^{-1}(\alpha)\right|}{(\beta-\alpha)^{n}} \cdot \frac{\beta-\alpha}{2} \\
& =\frac{C}{2} \cdot \frac{\left|f \circ v^{-1}(\beta)-f \circ v^{-1}(\alpha)\right|}{(\beta-\alpha)^{n-1}} \leq C \cdot \varepsilon \cdot\left(\frac{\alpha+\beta}{2}-\alpha\right)
\end{aligned}
$$


for $i=1,2$, and this inequality together with the equalities $l_{1}(\alpha)=l_{2}(\beta)=0$, and condition (iii) from Lemma 2.11, implies that $\left|F^{(n-1)}(t)\right|=\left|H_{\alpha, \beta}^{(n-1)}(t)\right|$ $\leq \min \left(l_{1}(t), l_{2}(t)\right) \leq C \varepsilon(t-x)$ for all $t \in(\alpha, \beta)$. To see this, we use the fact that if two continuous affine functions $a_{1}, a_{2}:[a, b] \rightarrow \mathbb{R}$ satisfy $a_{1}(a) \leq$ $a_{2}(a)$, and $a_{1}(b) \leq a_{2}(b)$, then $a_{1}(t) \leq a_{2}(t)$ for all $t \in[a, b]$. We apply this fact to $a_{1}(t)=l_{1}(t)$ for $t \in[\alpha,(\alpha+\beta) / 2]\left(\right.$ resp. $a_{1}(t)=l_{2}(t)$ for $t \in$ $[(\alpha+\beta) / 2, \beta])$, and $a_{2}(x)=C \varepsilon(t-x)$. Similarly for $(\alpha, \beta) \subset[x-\delta, x]$.

Let $\varepsilon>0$, and let $\delta>0$ be as in (3.39). We will show by induction (using (3.39)) that

$$
\left|F^{(i)}(t)\right| \leq C \varepsilon|t-x| \quad \text { for } t \in(x, x+\delta) \backslash v\left(K_{f}\right), i=1, \ldots, n-1 .
$$

Let $t^{\prime}:=\max \left(v\left(K_{f}\right) \cap[x, t]\right)$. Then (using the Mean Value Theorem and the fact that on $\left[t^{\prime}, t\right], F=H_{\alpha, \beta}$ for some $\left.\alpha, \beta\right)$ we obtain

$$
\begin{aligned}
\left|F^{(i)}(t)\right| & =\left|F^{(i)}(t)-F_{+}^{(i)}\left(t^{\prime}\right)\right| \leq\left|F^{(i+1)}\left(\xi_{i+1}\right)\right| \cdot\left|t-t^{\prime}\right| \\
& \leq \cdots \leq\left|F^{(n-1)}\left(\xi_{n-1}\right)\right| \cdot\left|t-t^{\prime}\right|^{n-1-i},
\end{aligned}
$$

where $\xi_{j} \in\left[t^{\prime}, t\right]$, and (3.40) easily follows.

Using (3.38), (3.40), and the fact that $F_{+}^{(i)}(\alpha)=H_{\alpha, \beta}^{(i)}(\alpha)=0$ for $i=$ $1, \ldots, n$, by induction we will obtain

$$
F^{(i)}(x)=0 \quad \text { for all } x \in v\left(K_{f}\right) \text { and } i=1, \ldots, n-1 .
$$

The case $i=1$ follows directly from (3.38). For $i=2, F^{\prime}(t)-F^{\prime}(x)=0$ provided $t \in v\left(K_{F}\right)$, and given $\varepsilon>0$, for $t \in(x-\delta, x+\delta) \backslash v\left(K_{f}\right)$ (where $\delta$ is chosen so that (3.38) and (3.40) hold) we have $\left|F^{\prime}(t)-F^{\prime}(x)\right|=\left|F^{\prime}(t)\right| \leq$ $C \varepsilon|t-x|$, and thus $F^{\prime \prime}(x)=0$. Similarly for higher $i$ 's.

To finish the proof of differentiability of $F$, it suffices to show that $F^{(n)}(x)=0$ for each $x \in v\left(K_{f}\right)$. Since $F^{(n-1)}(w)=0$ for all $w \in v\left(K_{f}\right)$, (3.39) together with (3.41) easily implies this assertion.

If $f$ is not constant on any interval, then (3.35) implies that $\lambda\left(K_{F}\right)=$ $\lambda\left(v\left(K_{f}\right)\right)=0$.

By (3.37) and (i) of Lemma 2.11, $F^{\prime}(x) \neq 0$ for all $x \in[c, d] \backslash K_{F}$. It follows that there exists a linear homeomorphism $\eta:[a, b] \rightarrow[c, d]$ which is onto. Now, it clearly suffices to put $\varphi:=F \circ \eta$.

Proposition 3.13. Let $f:[a, b] \rightarrow \mathbb{R}$ be a $C B V G_{1 / n}$ function. Then $f$ is Lebesgue equivalent to an $(n-1)$-times differentiable function $\phi$ such that $\phi^{(n-1)}(\cdot)$ is pointwise Lipschitz, $\phi^{(i)}(x)=0$ for all $x \in K_{\phi}, i=1, \ldots, n-1$, and $\phi^{\prime}(x) \neq 0$ whenever $x \in[a, b] \backslash K_{\phi}$. If $f$ is not constant on any interval, then $\lambda\left(K_{\phi}\right)=0$.

Proof. It is similar to the proof of Proposition 3.12, so we will only sketch it. If $f$ is not constant on any interval, then as in the proof of Proposition 3.12 we can assume that $\lambda\left(K_{f}\right)=0$. 
Let $\left(A_{m}\right)_{m \in \mathcal{M}}$ be the sets from Definition 3.3. If $\mathcal{M}$ is finite, then use $\tilde{A}_{m}=K_{f}$ and $\tilde{\mathcal{M}}=\mathbb{N}$ instead of $A_{m}$ and $\mathcal{M}$ (it is easy to see that in this case $\left.G V_{1 / n}\left(f, K_{f}, K_{f}\right)<\infty\right)$. Find a sequence $a_{m}>0$ such that

$$
\sum_{m \in \mathcal{M}} a_{m} \cdot G V_{1 / n}\left(f, A_{m} \cup\{a, b\}, K_{f}\right)<\infty .
$$

For $x \in[a, b] \cap K_{f}$ define $v(x)=x+\sum_{m \in \mathcal{M}} a_{m} \cdot\left(v_{m}(x)-\tilde{v}_{m}(x)\right)$, where $v_{m}$ (resp. $\tilde{v}_{m}$ ) come from Lemma 3.10 (resp. Lemma 3.11) applied to $f$ and $\delta=b-a$ (since it is easy to see that $\overline{G V_{1 / n}^{(b-a)}}\left(f, A_{m}, K_{f}\right) \leq 2 G V_{1 / n}\left(f, A_{m} \cup\right.$ $\left.\{a, b\}, K_{f}\right)$; see also (3.3)). As in the proof of Proposition 3.12, $v$ is a continuous strictly increasing function. Moreover, for each $x \in K_{f}$ there exists $m \in \mathcal{M}$ such that

$$
|f(y)-f(z)| \leq C_{m}|v(z)-v(y)|^{n-1}|v(z)-v(x)|
$$

for all $y, z \in K_{f}$ with $x \leq y<z$ or $z<y \leq x$. This follows from (3.24) and (3.33) just as (3.36) in the proof of Proposition 3.12.

Define $F:[a, b] \rightarrow \mathbb{R}$ as

$$
F(x):= \begin{cases}f \circ v^{-1}(x) & \text { for } x \in v\left(K_{f}\right), \\ H_{\alpha, \beta}(x) & \text { for } x \in(\alpha, \beta),\end{cases}
$$

whenever $(\alpha, \beta)$ is an interval contiguous to $v\left(K_{f}\right)$ in $[c, d]$, and $H=H_{\alpha, \beta}$ is is chosen by applying Lemma 2.11 to $\alpha, \beta, A=f \circ v^{-1}(\alpha), B=f \circ v^{-1}(\beta)$. Then $F$ is $n$-times differentiable at all $x \in[c, d] \backslash v\left(K_{f}\right)$. It remains to show that $F^{(i)}(x)=0$ for all $x \in v\left(K_{f}\right), i=1, \ldots, n-1$, and that $F^{(n-1)}$ is pointwise Lipschitz at all points of $v\left(K_{f}\right)$.

From (3.42), for each $x \in v\left(K_{f}\right)$ there exists $C_{x}>0$ such that

$$
|F(y)-F(z)| \leq C_{x}|z-y|^{n-1}|z-x|
$$

for $y, z \in v\left(K_{f}\right)$ with $z<y \leq x$ or $x \leq y<z$. This yields

$$
\left|F^{(n-1)}(t)\right| \leq C_{x}^{\prime}|t-x|
$$

for all $t \in[c, d] \backslash v\left(K_{f}\right)$. By induction, $F^{(i)}(x)=0$ for all $i=1, \ldots, n-1$ (since (3.44) together with (3.45) implies that $F^{\prime}(x)=0$, and then (3.45) easily implies that $F^{(i)}(x)=0$ for $i=2, \ldots, n-1$ ). Now (3.45) (together with the fact that $F^{(n-1)}(x)=0$ for each $x \in v\left(K_{f}\right)$ ) easily implies that $F^{(n-1)}$ is pointwise Lipschitz at all points of $v\left(K_{f}\right)$.

If $f$ is not constant on any interval, then as in Proposition 3.12, we establish that $\lambda\left(K_{F}\right)=\lambda\left(v\left(K_{f}\right)\right)=0$.

By (3.43) and (i) of Lemma 2.11, $F^{\prime}(x) \neq 0$ for all $x \in[c, d] \backslash K_{F}$. It follows that there exists a linear homeomorphism $\eta:[a, b] \rightarrow[c, d]$ which is onto. Now, it clearly suffices to put $\phi:=F \circ \eta$. 
4. Main result. The case $n=2$ is handled in [5]. The following main theorem gives a slightly different characterization in that case (see Introduction).

Theorem 4.1. Let $f:[a, b] \rightarrow \mathbb{R}$ be continuous and $n \in \mathbb{N}, n \geq 2$. Then the following are equivalent:

(i) $f$ is Lebesgue equivalent to a function $g$ which is $n$-times differentiable.

(ii) $f$ is Lebesgue equivalent to a function $g$ which is n-times differentiable and such that $g^{(i)}(x)=0$ whenever $i \in\{1, \ldots, n\}$ and $x \in K_{g}$, and $g^{\prime}(x) \neq 0$ whenever $x \in[a, b] \backslash K_{g}$.

(iii) $f$ is Lebesgue equivalent to a function $g$ which is $(n-1)$-times differentiable and such that $g^{(n-1)}(\cdot)$ is pointwise Lipschitz.

(iv) $f$ is $\overline{S B V G}_{1 / n}$.

(v) $f$ is $C B V G_{1 / n}$.

(vi) $f$ is $S B V G_{1 / n}$.

Proof. The implications (ii) $\Rightarrow($ i) and (i) $\Rightarrow$ (iii) are trivial. The implications (iii) $\Rightarrow$ (iv) and (iii) $\Rightarrow$ (v) follow from Lemma 3.9. The implication (vi) $\Rightarrow$ (ii) follows from Proposition 3.12, and (v) $\Rightarrow$ (iii) from Proposition 3.13. Finally, (i) $\Rightarrow$ (vi) follows from Lemmata 3.9 and 3.6 ; and (iv) $\Rightarrow$ (vi) from Lemma 3.6.

We have the following corollary:

Corollary 4.2. Let $f:[a, b] \rightarrow \mathbb{R}, n \geq 2, n \in \mathbb{N}$. Then $f$ is $C B V G_{1 / n}$ if and only if $f$ is $S B V G_{1 / n}$.

The following example shows that for each $n \geq 2$ there exists a continuous function $f:[0,1] \rightarrow \mathbb{R}$ that is $C B V G_{1 / n}$ (and thus is Lebesgue equivalent to an $n$-times differentiable function by Theorem 4.1$)$, but $V_{1 / n}\left(f, K_{f}\right)=\infty$ (and thus $f$ is not Lebesgue equivalent to any $C^{n}$ function by the results of [11]). It is a simplified version of [11, Example 8.3].

EXAMPLE 4.3. Let $n \geq 2$ be an integer. Let $a_{m} \subset(0,1)$ be such that $a_{m} \downarrow 0$. Define $f\left(a_{2 m}\right)=m^{-n}$ and $f(2 m-1)=0$ for all $m=1,2, \ldots$, $f(0)=f(1)=0$, and extend $f$ onto $[0,1]$ so that it is continuous and affine on each interval contiguous to $K=\{0,1\} \cup\left\{a_{m}: m \in \mathbb{N}\right\}$. Then $K_{f}=K$, $f$ is $C B V G_{1 / n}$, but $V_{1 / n}\left(f, K_{f}\right)=\infty$.

Proof. Obviously, $f$ is continuous, has bounded variation, and we have $V_{1 /(n-1)}\left(f, K_{f}\right)<\infty$. Also, it is easy to see that $f$ is $C B V G_{1 / n}$ (using $A_{1}=\{0,1\}$ and $A_{m}=\left\{a_{2 m-2}, a_{2 m-3}\right\}$ for $\left.m=2,3, \ldots\right)$. On the other hand,

$$
V_{1 / n}\left(f, K_{f}\right) \geq \sum_{m \in \mathbb{N}}\left|f\left(a_{2 m}\right)-f\left(a_{2 m-1}\right)\right|^{1 / n}=\sum_{m} \frac{1}{m}=+\infty .
$$


The following theorem characterizes the situation when we require the first derivative to be nonzero almost everywhere.

Theorem 4.4. Let $f:[a, b] \rightarrow \mathbb{R}$ be continuous and $n \in \mathbb{N}, n \geq 2$. Then the following are equivalent:

(i) $f$ is Lebesgue equivalent to a function $g$ which is $n$-times differentiable and such that $g^{\prime}(x) \neq 0$ for a.e. $x \in[a, b]$.

(ii) $f$ is Lebesgue equivalent to a function $g$ which is n-times differentiable and such that $g^{(i)}(x)=0$ whenever $i \in\{1, \ldots, n\}$ and $x \in K_{g}$, $g^{\prime}(x) \neq 0$ whenever $x \in[a, b] \backslash K_{g}$, and $\lambda\left(K_{g}\right)=0$.

(iii) $f$ is Lebesgue equivalent to a function $g$ which is $(n-1)$-times differentiable and such that $g^{(n-1)}(\cdot)$ is pointwise Lipschitz, and $g^{\prime}(x) \neq 0$ for a.e. $x \in[a, b]$.

(iv) $f$ is $\overline{S B V G}_{1 / n}$ and $f$ is not constant on any interval.

(v) $f$ is $C B V G_{1 / n}$ and $f$ is not constant on any interval.

(vi) $f$ is $S B V G_{1 / n}$ and $f$ is not constant on any interval.

Proof. The proof is similar to the proof of Theorem 4.1 while we also use the fact that $g^{\prime}(x) \neq 0$ for a.e. $x \in[a, b]$ implies that $g$ is not constant on any interval, the fact that being nonconstant on any interval is invariant with respect to the Lebesgue equivalence, and the corresponding assertions in Propositions 3.12 and 3.13.

5. Generalized Zahorski Lemma. Our methods yield the following theorem, which can be viewed as a generalization of Zahorski's Lemma; see e.g. [15] or [10, p. 27].

Theorem 5.1. Let $K \subset[a, b]$ be a closed set and $n \in \mathbb{N}, n \geq 2$. Then the following are equivalent:

(i) There exists an $n$-times differentiable homeomorphism $h$ of $[a, b]$ onto itself such that

$$
K=h\left(\left\{x \in[a, b]: h^{(i)}(x)=0 \forall i=1, \ldots, n\right\}\right) .
$$

(ii) $V_{1 /(n-1)}(\mathrm{id}, K)<\infty$ and there exist closed sets $A_{m} \subset[a, b](m \in$ $\mathcal{M} \subset \mathbb{N})$ such that $K=\bigcup_{m} A_{m}$ and $G V_{1 / n}\left(\mathrm{id}, A_{m}, K\right)<\infty$ for all $m \in \mathcal{M}$.

(iii) $V_{1 /(n-1)}(\mathrm{id}, K)<\infty$ and there exist closed sets $A_{m} \subset[a, b]$ and numbers $\delta_{m}>0(m \in \mathbb{N})$ such that $K=\bigcup_{m} A_{m}, A_{m} \subset A_{m+1}$ for all $m \in \mathbb{N}$, and $\lim _{m \rightarrow \infty} \overline{G V}_{1 / n}^{\delta_{m}}\left(\mathrm{id}, A_{m}, K\right)=0$.

Proof. Since the proof is similar to the considerations above, we will only sketch it. Without any loss of generality, we can assume that $\{a, b\}$ $\subset K$. The proof of $(\mathrm{i}) \Rightarrow(\mathrm{ii})$ is similar to the proof that every $(n-1)$-times 
differentiable function $f$ such that $f^{(n-1)}$ is pointwise Lipschitz, is $C B V G_{1 / n}$ (see Lemma 3.9).

To prove that (ii) $\Rightarrow$ (iii), note that an argument similar to the proof of Proposition 3.13 shows that there exists a homeomorphism $\varphi$ of $[a, b]$ onto itself which is $(n-1)$-times differentiable with $\varphi^{(n-1)}$ being pointwise Lipschitz, and such that

$$
K=\varphi\left(\left\{x \in[a, b]: \varphi^{(i)}(x)=0 \text { for all } i=1, \ldots, n-1\right\}\right) .
$$

By the argument of the proof of Lemma 3.9, we find a decomposition $\tilde{A}_{m}^{k}$ with some $\tilde{\delta}_{m}^{k}>0$ for $\varphi^{-1}(K)$ such that $\tilde{A}_{m}^{k} \subset \tilde{A}_{m+1}^{k}$ for all $m, k \in \mathbb{N}$ and

$$
\lim _{m \rightarrow \infty} \overline{G V_{1 / n}^{\delta_{m}^{k}}}\left(\mathrm{id}, \tilde{A}_{m}^{k}, \varphi^{-1}(K)\right)=0
$$

for each $k$, and then using the diagonal argument from Lemma 3.6, we find $\tilde{A}_{m}$ and $\tilde{\delta}_{m}$ such that $\varphi^{-1}(K)=\bigcup_{m} \tilde{A}_{m}$ and

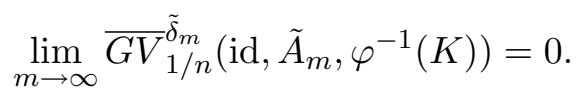

Now, we put $A_{m}:=\varphi\left(\tilde{A}_{m}\right)$, and find suitable $\delta_{m}>0$ using the uniform continuity of $\varphi^{-1}$. This shows that (iii) holds.

Finally, to show that (iii) $\Rightarrow($ i), one can use a construction similar to the one in the proof of Proposition 3.12.

REMARK 5.2. It is also not very difficult to see that in the previous theorem, we can replace (i) with

(i') There exists an $n$-times differentiable homeomorphism $h$ of $[a, b]$ onto itself such that $h^{-1}$ is absolutely continuous, $h^{-1}(K)=0$, and

$$
K=h\left(\left\{x \in[a, b]: h^{(i)}(x)=0 \forall i=1, \ldots, n\right\}\right) .
$$

The proof uses ideas from the proof of Theorem 4.4. Let us only remark that it is well known that $h^{-1}$ can be taken absolutely continuous in the classical Zahorski Lemma; see e.g. [1].

Acknowledgments. The author would like to thank Professor Luděk Zajiček for several remarks that led to considerable improvements of the presentation.

The author was supported in part by ISF.

\section{References}

[1] A. Bruckner, Differentiation of Real Functions, 2nd ed., CRM Monogr. Ser. 5, Amer. Math. Soc., Providence, 1994.

[2] A. M. Bruckner and C. Goffman, Differentiability through change of variables, Proc. Amer. Math. Soc. 61 (1976), 235-241. 
[3] L. Cesari, Rectifiable curves and the Weierstrass integral, Amer. Math. Monthly 65 (1958), 485-500.

[4] G. Choquet, Application des propriétés descriptives de la fonction contingent à la théorie des fonctions de variable réelle et à la géométrie différentielle des variétés cartésiennes, J. Math. Pures Appl. (9) 26 (1947), 115-226.

[5] J. Duda, Second order differentiability of paths via a generalized $\frac{1}{2}$-variation, J. Math. Anal. Appl. 338 (2008), 628-638.

[6] J. Duda and L. Zajíček, Curves in Banach spaces - differentiability via homeomorphisms, Rocky Mountain J. Math. 37 (2007), 1493-1525.

[7] -, -, Curves in Banach spaces which allow a $C^{2}$ parametrization, submitted.

[8] -, - Curves in Banach spaces which allow a parametrization with finite convexity, work in progress.

[9] R. J. Fleissner and J. A. Foran, A note on differentiable functions, Proc. Amer. Math. Soc. 69 (1978), 56.

[10] C. Goffman, T. Nishiura and D. Waterman, Homeomorphisms in Analysis, Math. Surveys Monogr. 54, Amer. Math. Soc., Providence, RI, 1997.

[11] M. Laczkovich and D. Preiss, $\alpha$-variation and transformation into $C^{n}$ functions, Indiana Univ. Math. J. 34 (1985), 405-424.

[12] V. V. Lebedev, Homeomorphisms of a segment and smoothness of a function, Mat. Zametki 40 (1986), 364-373, 429 (in Russian).

[13] S. Saks, Theory of the Integral, Monografie Mat. 7, Stechert, New York, 1937.

[14] G. P. Tolstov, Curves allowing a differentiable parametric representation, Uspekhi Mat. Nauk (N.S.) 6 (1951), no. 3, 135-152.

[15] Z. Zahorski, On Jordan curves possessing a tangent at every point, Mat. Sb. (N.S.) 22 (64) (1948), 3-26 (in Russian).

Department of Mathematics

Weizmann Institute of Science

Rehovot 76100, Israel
Current address:

PIRA Energy Group

3 Park Ave FL 26

New York, NY 10016, U.S.A.

E-mail: jakub.duda@gmail.com

Received 18 December 2005;

in revised form 28 July 2009 